MIT-CTP-2527

\title{
NEW MODULI SPACES FROM STRING BACKGROUND INDEPENDENCE CONSISTENCY CONDITIONS
}

\author{
BARTON ZWIEBACH ${ }^{\star}$ \\ Center for Theoretical Physics, \\ Laboratory of Nuclear Science \\ and Department of Physics \\ Massachusetts Institute of Technology \\ Cambridge, Massachusetts 02139, U.S.A.
}

\begin{abstract}
In string field theory an infinitesimal background deformation is implemented as a canonical transformation whose hamiltonian function is defined by moduli spaces of punctured Riemann surfaces having one special puncture. We show that the consistency conditions associated to the commutator of two deformations are implemented by virtue of the existence of moduli spaces of punctured surfaces with two special punctures. The spaces are antisymmetric under the exchange of the special punctures, and satisfy recursion relations relating them to moduli spaces with one special puncture and to string vertices. We develop the theory of moduli spaces of surfaces with arbitrary number of special punctures and indicate their relevance to the construction of a string field theory that makes no reference to a conformal background. Our results also imply a partial antibracket cohomology theorem for the string action.
\end{abstract}

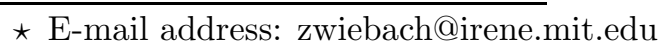

Supported in part by D.O.E. cooperative agreement DE-FC02-94ER40818. 


\section{Introduction and Summary}

Finding the conceptual framework for string theory is an outstanding open problem of great relevance. One way to search for this framework is to develop and extend as far as is possible the present formulation of string field theory. This present formulation uses explicitly a chosen conformal background and is not manifestly independent of this choice. Nevertheless this admittedly incomplete string field theory has revealed a rather elegant structure. The mathematical framework of the present-day field theory of strings is by now fairly clear. It contains an ingredient from Riemann surfaces and an ingredient from conformal theory, with similar and related structures existing in both.

Consider first the Riemann surface ingredient. For moduli spaces of punctured surfaces, the natural operation of cutting disks around punctures and sewing the resulting boundaries with a twist allow one to define the antibracket $\{$,$\} of two moduli spaces of surfaces and a$ delta operation $\Delta$ on a moduli space of surfaces. These operations satisfy the axioms of the Batalin-Vilkovisky (BV) antibracket and delta operation. Moreover, the sets of moduli spaces that define string vertices can be grouped into an element called $\mathcal{V}$, and the condition that the corresponding Feynman graphs generate a cover of all moduli spaces of Riemann surfaces implies that $\mathcal{V}$ can be used to define a cohomology class for the operator $\partial+\Delta$, where $\partial$ is the boundary operator on moduli spaces of surfaces.

Consider now the conformal theory ingredient. A suitable conformal field theory furnishes a vector space $\widehat{\mathcal{H}}$ spanned by all local operators, and equipped with an antibracket and a delta operation defined on the functions on $\widehat{\mathcal{H}}$. Moreover it furnishes an odd quadratic hamiltonian $Q$ satisfying $\{Q, Q\}=0$ arising from the BRST operator on $\widehat{\mathcal{H}}$. There is, moreover, a map $f$ from moduli spaces of surfaces to functions on $\widehat{\mathcal{H}}$. This map induces a homomorphism between the BV structure on moduli spaces and the BV structure on $\widehat{\mathcal{H}}$, with the boundary operator $\partial$ becoming the BRST hamiltonian $Q$. Finally the string action $S$ is simply given as $S=Q+f(\mathcal{V})$, and is a function that manifestly satisfies the BV master equation, thus defining a gauge invariant action that can be quantized consistently.

The above viewpoint on string field theory was explained precisely in Ref.[1] and was obtained while investigating the problem of background independence [ 2,3,4]. There are certainly other ways of viewing string field theory, where one emphasizes other aspects of the algebraic structure or other aspects of the geometrical foundation of the theory [ 5,6,7]. For related matters see also Refs.[ 8,9,10], and for recent discussion see Refs.[ 11,12]. 
Since present-day string field theory is not manifestly independent of the choice of background needed for its formulation, the above structure cannot be expected to be the final word. In fact, the analysis of background independence of Refs.[2,3] brought into the open an operator $\mathcal{K}$ which acting on a Riemann surface adds one special puncture throughout the surface minus the unit disks around the punctures (the disks that define the local coordinates around the punctures). This operation is intimately related, at the level of spaces of conformal theories, to the operation of covariant differentiation using a particular connection $[13,14,15,16,17]$. In addition to the operator $\mathcal{K}$, a new family of moduli spaces of Riemann surfaces was introduced, spaces where the surfaces have one special puncture in addition to the ordinary punctures. These spaces, called $\mathcal{B}^{1}$ spaces (our earlier notation used no superscript), have a real dimensionality that exceeds by one that of the corresponding moduli space with only ordinary punctures. More precisely, the moduli space $\mathcal{B}_{g, n-1}^{1}$ having $(n-1)$ ordinary punctures and one special one, has dimension equal to that of $\mathcal{M}_{g, n}$ plus one (that is $6 g-6+2 n+1)$. Roughly speaking the $\mathcal{B}_{g, n-1}^{1}$ space interpolates between the string vertex $\mathcal{V}_{g, n}$ where one of the punctures is considered special, and $\mathcal{K} \mathcal{V}_{g, n-1}$ which is the "vertex" obtained by adding one special puncture via $\mathcal{K}$ to a vertex with one less puncture.

The $\mathcal{B}^{1}$ spaces were the Riemann surface ingredient for the proof of background independence of string field theory. Inserting a marginal operator $\widehat{\mathcal{O}}_{\mu}$ in the special puncture, the $\mathcal{B}^{1}$ spaces define a hamiltonian function $B_{\mu}$ (formerly called $U_{\mu}$ ) that by canonical action via the antibracket generates a change of string background. Given that they play such prominent role in the proof of local background independence it was suggested in Ref.[3] that $\mathcal{B}^{1}$ spaces could also play a role in the construction that makes background independence manifest.

Here we indicate a natural way the suggestion can be realized if we attempt to construct a string field theory around a non-conformal background. A string field theory written using a non-conformal background would represent concrete progress since the main problem with the present formulation is not that we use a background to write the theory, but rather the fact that the background needs to be conformal. The algebraic structure of string theory formulated around non-conformal backgrounds was explored by indirect means in Ref.[7] sect 4.5. This structure requires a special state $F$ of ghost number three whose vanishing would imply that we are again considering a conformal theory. This special string field $F$ modifies significantly the identities that must be satisfied by the string vertices. We now recall that ordinary closed string fields are of ghost number two and ghost number conservation requires correlations of ordinary closed string fields to be integrated on moduli spaces of standard dimensionality. 
Being of ghost number three in order to couple $F$ to ordinary string fields we require moduli spaces of real dimension one higher than the standard dimension. As mentioned in the previous paragraph $\mathcal{B}^{1}$ spaces have precisely this property! The way to use the $\mathcal{B}^{1}$ spaces to construct explicitly an action representing string theory around non-conformal backgrounds will be the subject of a forthcoming publication [18].

It can be guessed, however, that the presently known $\mathcal{B}^{1}$ spaces cannot be the complete story. Moduli spaces whose surfaces have only one special puncture clearly represent first order perturbations of the background. We should expect that moduli spaces $\mathcal{B}^{2}, \mathcal{B}^{3}, \cdots$ of surfaces with two, with three, in fact, with all numbers of special punctures should be relevant [18]. We must therefore learn how to deal with moduli spaces whose surfaces contain more than one special puncture, and this is the main goal of the present paper. The case of two special punctures brings in most of the new features of the problem, and therefore much of our work here will have to do with this case. Luckily, this case need not be investigated in the difficult context of constructing a more general string field theory.

In this paper we show that moduli spaces $\mathcal{B}^{2}$ of surfaces with two special punctures make an appearance when we consider consistency conditions arising from the commutator of two infinitesimal background deformations. Arising from a commutator, $\mathcal{B}^{2}$ spaces should be antisymmetric under the exchange of the two special punctures. This is also in accord with our expectation for such spaces in a new formulation of string field theory; if we are to insert ghost number three grassmann odd $F$ states on every special puncture, moduli spaces of surfaces with special punctures must be odd under the exchange of any pair of special punctures. This antisymmetry brings about a number of simplifications.

When we examine the consistency conditions for background independence we obtain a condition of the form $\left\{S, H_{\mu \nu}\right\}=0$, where $S$ is the string action. $H_{\mu \nu}$ is an object built from covariant derivatives of the hamiltonian $B_{\mu}$ generating a background deformation in the direction of the marginal operator $\widehat{\mathcal{O}}_{\mu}$, and from the curvature $R_{\mu \nu}$ of a theory space connection. While it is clear from our arguments that the consistency condition is satisfied, we demand a stronger result, we require that $H_{\mu \nu}=\left\{S, B_{\mu \nu}\right\}$, where $B_{\mu \nu}$ is a hamiltonian to be determined. It then follows that $\left\{S,\left\{S, B_{\mu \nu}\right\}\right\}=0$ by virtue of the Jacobi identity and the master equation $\{S, S\}=0$. The existence of $B_{\mu \nu}$ amounts to a cohomology theorem for the

* In the analysis of background independence of Refs.[2,3] one inserted a grassman even marginal state on the special puncture of the $\mathcal{B}$ spaces. The construction of hamiltonians for large background deformations may require moduli spaces symmetric under the exchange of the special punctures. 
string action $S$, in this case saying that the $S$-closed function $H_{\mu \nu}$ is $S$ exact. Our goal is not only to show that the hamiltonian $B_{\mu \nu}$ exists, but also to show that it is simply a hamiltonian associated to moduli spaces $\mathcal{B}^{2}$ of surfaces having two special punctures. This is certainly not manifest since the object $H_{\mu \nu}$ appears to involve the theory space connection in a nontrivial way.

Our results, apart from their significance on the context of building a more general string field theory, represent some progress towards developing a complete understanding of the antibracket cohomology defined by the string action $S$. In fact, even the existence (shown in [2]) of the hamiltonian $B_{\mu}$ generating the infinitesimal string background deformations can be viewed as a cohomology theorem. Cohomology theorems are useful to understand issues of uniqueness, anomalies, and renormalization. Complete antibracket cohomology theorems are available for theories of nonabelian gauge fields and gravitation [19].

In order to understand the moduli space origin of the hamiltonian $B_{\mu \nu}$ we must develop a little the geometrical understanding of connections achieved in Ref.[ 15]. In particular we show that the reference connection $\Gamma$ (called $\widehat{\Gamma}$ in [15]) is such that the antisymmetric combination of covariant derivatives $D_{\mu}\left|\widehat{\mathcal{O}}_{\nu}\right\rangle-D_{\nu}\left|\widehat{\mathcal{O}}_{\mu}\right\rangle$ is simply related to a moduli space $\mathcal{T}_{1}^{2}$ of spheres with one ordinary puncture and two special punctures having the states $\widehat{\mathcal{O}}_{\mu}$ and $\widehat{\mathcal{O}}_{\nu}$ inserted in them. Moreover, we give a simple proof that the connection $\Gamma$ has zero curvature.

We generalize our earlier definition of the antibracket of two moduli spaces of surfaces to include the case when the moduli spaces are comprised of surfaces with special punctures. We introduce a new operator $\mathcal{I}$ whose effect is to turn an ordinary puncture into a special puncture preserving the antisymmetry of all the special punctures. Since moduli spaces are symmetric under the exchange of ordinary punctures $\mathcal{I}^{2}=0$ follows because repeated action by $\mathcal{I}$ will turn two ordinary punctures special. We also find a suitable definition for $\mathcal{K}$ acting on surfaces having special punctures. The operator $\mathcal{K}$ will insert a new puncture on the surface minus the unit disks around the ordinary punctures; it ignores the presence of the special punctures. The collisions between the old special punctures and the moving special puncture are rendered harmless by the antisymmetry condition. Moreover we show that $\mathcal{K}^{2}=0$, a property that is intimately related the zero curvature property of the connection $\Gamma$. We develop a series of identities relating the operators $\mathcal{K}, \mathcal{I}$ and the antibracket.

Equipped with the above tools we can show that the hamiltonian $B_{\mu \nu}$ is indeed defined by moduli spaces of surfaces with two special punctures, moduli spaces denoted collectively 
as $\mathcal{B}^{2}=\sum_{n \geq 1} \mathcal{B}_{n}^{2}$, where $\mathcal{B}_{n}^{2}$ is a moduli space of spheres with two special punctures and $n$ ordinary punctures. Similarly, we write $\mathcal{B}^{1}=\sum_{n \geq 2} \mathcal{B}_{n}^{1}$ where $\mathcal{B}_{n}^{1}$ is a moduli space of spheres with one special puncture and $n$ ordinary punctures. The equations that constrain $\mathcal{B}^{2}$ take the form of recursion relations involving the $\mathcal{B}^{1}$ spaces and the string vertices $\mathcal{V}$. They read

$$
\partial \mathcal{B}^{2}=\mathcal{T}_{1}^{2}+(\mathcal{K}-\mathcal{I}) \mathcal{B}^{1}-\frac{1}{2}\left\{\mathcal{B}^{1}, \mathcal{B}^{1}\right\}-\left\{\mathcal{V}, \mathcal{B}^{2}\right\}
$$

We prove the consistency of the above equations by showing that the right hand side is annihilated by the boundary operator $\partial$. We then give a recursive construction of the $\mathcal{B}^{2}$ spaces.

All our work will concern only the classical string action $S$. No particular difficulties are expected for the extension to the full quantum master action, apart from the usual subtleties associated to vacuum vertices, particularly at genus one. While we give here the general framework to deal with moduli spaces having any number of special punctures, the explicit conditions defining useful moduli spaces $\mathcal{B}^{3}, \mathcal{B}^{4}, \cdots$, will be given in Ref.[18]. As the analysis of the present work suggests, they can be obtained as higher cohomology theorems resulting from multiple commutators of background deformations.

This paper is organized as follows. In sect. 2 we review some necessary results on connections on spaces of conformal field theories and covariant derivatives. We also recall the main properties of the string vertices $\mathcal{V}$ and the $\mathcal{B}^{1}$ spaces. In sect. 3 we derive and examine the consistency conditions arising from the commutator of two infinitesimal background deformations. In sect. 4 we develop further our understanding of the canonical connection $\Gamma$; we define the space of surfaces $\mathcal{T}_{1}^{2}$ related to the computation of $D_{\mu}\left|\widehat{\mathcal{O}}_{\nu}\right\rangle-D_{\nu}\left|\widehat{\mathcal{O}}_{\mu}\right\rangle$, and study its properties. We also simplify the computation of curvature. In sect.5 we define our moduli spaces of surfaces with one or more special punctures and introduce the relevant antibracket, $\mathcal{K}$ operator and $\mathcal{I}$ operator. We also discuss the homomorphism from moduli spaces to functions on the state space of the conformal theory. In sect. 6 we use our previous developments to show that the consistency conditions can be satisfied through the existence of suitable moduli spaces $\mathcal{B}^{2}$ of surfaces with two special punctures. Sect.7 is devoted to the recursive construction of the $\mathcal{B}^{2}$ spaces. 


\section{Review and Notation}

In this section we will review some of the basic facts required in the present work. Most of these facts were explained in detail in earlier publications so we will not offer derivations. The reader should have no problem in deriving the quoted results using the original papers. We begin with connections, and later turn to moduli spaces of Riemann surfaces, in particular we consider the string vertices $\mathcal{V}$ and the $\mathcal{B}^{1}$ spaces.

\subsection{ConneCtions on SPACES OF CONFORMal THEORIES}

Given a space $M$ of conformal theories, with coordinates $x^{\mu}$ we construct a vector bundle over $M$ by considering at every point of $M$ a vector space spanned by a basis $\left|\Phi_{i}\right\rangle$ for the states of the conformal theory. We use $\psi^{i}$ to denote a coordinate on the vector space. A connection on this vector bundle is defined by coefficients $\Gamma_{\mu i}^{j}$, and covariant derivatives of functions on this bundle are defined as

$$
D_{\mu}(\Gamma) A \equiv \partial_{\mu} A-\frac{\partial_{r} A}{\partial \psi^{i}} \Gamma_{\mu j}^{i} \psi^{j}
$$

It then follows that

$$
\left[D_{\mu}, D_{\nu}\right] A=-\frac{\partial_{r} A}{\partial \psi^{i}} R_{\mu \nu j}^{i} \psi^{j}=-\frac{\partial_{r} A}{\partial \psi} R_{\mu \nu} \psi
$$

where in the last step we use matrix notation $R_{\mu \nu}=\left|\Phi_{j}\right\rangle R_{\mu \nu i}^{j}\left\langle\Phi^{j}\right|$. We have

$$
R_{\mu \nu}=\partial_{\mu} \Gamma_{\nu}-\partial_{\nu} \Gamma_{\mu}+\Gamma_{\mu} \Gamma_{\nu}-\Gamma_{\nu} \Gamma_{\mu}
$$

where we also use matrix notation for the connection $\Gamma_{\mu} \equiv\left|\Phi_{j}\right\rangle \Gamma_{\mu i}^{j}\left\langle\Phi^{j}\right|$. The vector bundles we are interested in have a symplectic form

$$
\left\langle\omega_{12}\right|=-{ }_{1}\left\langle\Phi^{i}\right| \omega_{i j}(x)_{2}\left\langle\Phi^{j}\right|=\left\langle R_{12}^{\prime}\right| c_{0}^{-(2)},
$$

which satisfies $\left\langle\omega_{12}\right|=-\left\langle\omega_{21}\right|$, and whose inverse $\left|S_{12}\right\rangle$ satisfies $\left|S_{12}\right\rangle=\left|S_{21}\right\rangle$. Given the symplectic form we define an antibracket

$$
\{A, B\}=\frac{\partial_{r} A}{\partial \psi^{i}} \omega^{i j} \frac{\partial_{l} B}{\partial \psi^{j}},
$$

with standard exchange property $\{A, B\}=-(-)^{(A+1)(B+1)}\{B, A\}$ and satisfying the Jacobi 
identity

$$
(-)^{(A+1)(C+1)}\{\{A, B\}, C\}+\operatorname{Cyclic}(A \rightarrow B \rightarrow C)=0 .
$$

It is sometimes convenient to use explicit string field notation $|\Psi\rangle \equiv\left|\Phi_{i}\right\rangle \psi^{i}$. In this notation (2.1) reads

$$
D_{\mu} A=\partial_{\mu} A-\frac{\partial A}{\partial|\Psi\rangle} \Gamma_{\mu}|\Psi\rangle .
$$

The covariant derivative of functions of the type $\langle A \mid \Psi\rangle \cdots|\Psi\rangle$ defined by tensor sections $\langle A|$ is given by

$$
D_{\mu}\langle A \mid \Psi\rangle \cdots|\Psi\rangle=\left(D_{\mu}\langle A|\right)|\Psi\rangle \cdots|\Psi\rangle
$$

where covariant derivatives of sections are simply given by

$$
D_{\mu}(\Gamma)\langle A|=\partial_{\mu}\langle A|-\sum_{n}\langle A| \Gamma_{\mu}^{(n)},
$$

with the label $n$ referring to a state space in the tensor section. The antibracket can also be written as

$$
\{A, B\}=(-)^{B+1} \frac{\partial A}{\partial|\Psi\rangle} \frac{\partial B}{\partial|\Psi\rangle}|S\rangle .
$$

The connections we will be dealing with are symplectic; namely they satisfy $D_{\mu}(\Gamma)\left\langle\omega_{12}\right|=0$. It then follows that covariant derivatives act as derivations of the antibracket

$$
D_{\mu}\{A, B\}=\left\{D_{\mu} A, B\right\}+\left\{A, D_{\mu} B\right\}
$$

The curvature of a symplectic connection is hamiltonian, namely, the right hand side of (2.2) can be reproduced by canonical action. Indeed, using $\left\langle\omega_{12}\right|\left(R_{\mu \nu}^{(1)}+R_{\mu \nu}^{(2)}\right)=0$, which follows by commutator action on the symplectic form, one can readily show that

$$
\left[D_{\mu}, D_{\nu}\right] A=-\left\{A, R_{\mu \nu}\right\}
$$

where the curvature hamiltonian $R_{\mu \nu}$, denoted with a slight abuse of notation with the same symbol as the curvature matrix, is given by

$$
R_{\mu \nu}=-\frac{1}{2}\left\langle\omega_{12}\left|R_{\mu \nu}^{(2)}\right| \Psi\right\rangle|\Psi\rangle
$$

Having reviewed general facts about connections let us recall some properties of the particular connection we shall be using here. This is the connection $\widehat{\Gamma}$ of Ref.[15], anticipated 
in Refs[13,14,20]. This connection, denoted hereafter as $\Gamma$, is best described (in the spirit of [16]) by indicating how to take covariant derivatives. Let $\Sigma$ denote a punctured Riemann surface with punctures $P_{1}, \cdots P_{n}$, with non overlapping coordinate disks $D_{1}, \cdots D_{n}$, and let $\widehat{\Sigma}=\Sigma-\cup_{i} D_{i}$ denote the surface minus its unit disks. The covariant derivative of the surface state $\langle\Sigma|$ is given by

$$
D_{\mu}\langle\Sigma|=-\frac{1}{\pi} \int_{p \in \widehat{\Sigma}} d \mu(p)\left\langle\Sigma ; p \mid \mathcal{O}_{\mu}(p)\right\rangle .
$$

Here the measure of integration is unambiguous since the operator $\mathcal{O}_{\mu}$ is a dimension $(1,1)$ primary operator. The covariant derivatives of the Virasoro operators allow one to show that ([ 15], Eqn.(5.10))

$$
\Gamma_{\mu \nu}^{k}=\frac{1}{\pi} \frac{H_{\mu \nu}^{k} \delta_{0, s_{k}}}{2-\gamma_{k}}, \text { for } \gamma_{k} \neq 2,
$$

where $s_{k}$ and $\gamma_{k}$ denote the spin and total conformal dimension of the state $\left|\Phi_{k}\right\rangle$. Ref.[ 15] also discussed another connection $c_{\mu i}^{j}$, first introduced in the work of Sonoda [16]. For $\gamma_{k} \leq 2$ one can show that $\Gamma_{\mu \nu}^{k}=c_{\mu \nu}^{k}$ (Ref.[15], Eqn.(4.10)). This, together with the torsion analysis of Ref.[15] (see Eqns.(6.25-29)) which explained that $c_{\mu \nu}^{k}-c_{\nu \mu}^{k}=0$ for $\gamma_{k} \leq 2$, implies that

$$
\Gamma_{\mu \nu}^{k}-\Gamma_{\nu \mu}^{k}=0, \quad \text { for } \gamma_{k} \leq 2 .
$$

It may be possible to give a somewhat simpler derivation of this fact in the framework of [17].

\subsection{Moduli spaCes of STRING Vertices AND $\mathcal{B}$ SPACES}

The string vertices $\mathcal{V}=\sum_{n \geq 3} \mathcal{V}_{n}$ at genus zero satisfy the recursion relations (see, for example, Ref.[3] Eqn.(2.22))

$$
\partial \mathcal{V}+\frac{1}{2}\{\mathcal{V}, \mathcal{V}\}=0
$$

These recursion relations can be expressed as the fact that the operator $\delta_{\mathcal{V}}$ squares to zero:

$$
\delta_{\mathcal{V}}^{2}=0, \quad \text { with } \quad \delta_{\mathcal{V}} \equiv \partial+\{\mathcal{V},\}
$$

Moreover, we readily find that

$$
\delta_{\mathcal{V}} \mathcal{V}=\frac{1}{2}\{\mathcal{V}, \mathcal{V}\}
$$

It is useful to introduce a new piece of notation, an operator $\mathcal{I}$ that will be discussed in detail in sect.5. This operator makes one of the ordinary punctures of a surface special. Since 
our moduli spaces are symmetric under the exchange of labels of punctures on the surfaces, any puncture may be chosen. This operator acts as a derivation of the antibracket, and one readily verifies that

$$
\delta_{\mathcal{V}} \mathcal{I V}=0
$$

At genus zero, the interpolating $\mathcal{B}^{1}$ spaces of background independence (formerly called $\mathcal{B})$ have one special puncture, and exist for two or more ordinary punctures. We write $\mathcal{B}^{1} \equiv$ $\sum_{n \geq 2} \mathcal{B}_{n}^{1}$, where $n$ denores the number of ordinary punctures. Their recursion relations take the form (see, for example Ref.[21], Eqn.(3.18))

$$
\partial \mathcal{B}^{1}=\mathcal{V}_{3}^{\prime}+(\mathcal{K}-\mathcal{I}) \mathcal{V}-\left\{\mathcal{V}, \mathcal{B}^{1}\right\}
$$

The two lowest dimension cases here are

$$
\begin{aligned}
& \partial \mathcal{B}_{2}^{1}=\mathcal{V}_{3}^{\prime}-\mathcal{I V}_{3} \\
& \partial \mathcal{B}_{3}^{1}=\mathcal{K} \mathcal{V}_{3}-\mathcal{I V}_{4}-\left\{\mathcal{V}_{3}, \mathcal{B}_{2}^{1}\right\}
\end{aligned}
$$

The recursion relation (2.21) can be written more compactly using the operator $\delta_{\mathcal{V}}$ introduced in (2.18). We get

$$
\delta_{\mathcal{V}} \mathcal{B}^{1}=\mathcal{V}_{3}^{\prime}+(\mathcal{K}-\mathcal{I}) \mathcal{V}
$$

\section{Commutator of Background Deformations}

In this section we reconsider the equation which defining the hamiltonian that implements an infinitesimal background deformation. For simplicity we only consider the classical string field theory. The equation in question was derived in [2] and reads

$$
D_{\mu}(\Gamma) S=\left\{S, B_{\mu}\right\} .
$$

Local background independence at the classical level was established by finding the explicit hamiltonian $B_{\mu}$ (formerly called $U_{\mu}$ ) satisfying this equation. It was found that $B_{\mu}$ is given by

$$
B_{\mu}=B_{\mu}^{(2)}-f_{\mu}\left(\mathcal{B}^{1}\right),
$$

where $B_{\mu}^{(2)}$ is a quadratic hamiltonian implementing an $\widehat{\mathcal{O}}_{\mu}$ insertion. We will take a second covariant derivative of (3.1) and will show the existence of an $S$-closed function $H_{\mu \nu}$. We explain how the expected uniqueness of the string master action requires that $H_{\mu \nu}$ be $S$-trivial: $H_{\mu \nu}=\left\{S, B_{\mu \nu}\right\}$, with $B_{\mu \nu}$ a hamiltonian to be determined. 


\subsection{The COMmutator CONDitions}

Taking a second covariant derivative of Eqn.(3.1), and making the connection implicit we find

$$
D_{\mu} D_{\nu} S=\left\{\left\{S, B_{\mu}\right\}, B_{\nu}\right\}+\left\{S, D_{\mu} B_{\nu}\right\}
$$

where use was made of the fact that the connection is symplectic and therefore covariant derivatives act as derivations of the antibracket (see Eqn.(2.11)). We now form the commutator and find

$$
\left[D_{\mu}, D_{\nu}\right] S=\left\{S, D_{\mu} B_{\nu}-D_{\nu} B_{\mu}+\left\{B_{\mu}, B_{\nu}\right\}\right\}
$$

On the other hand the commutator is given by curvature $\left[D_{\mu}, D_{\nu}\right] S=-\left\{S, R_{\mu \nu}\right\}$ (see (2.12)), and therefore we find the consistency condition

$$
\left\{S, H_{\mu \nu}\right\}=0
$$

where the object $H_{\mu \nu}$ is given by

$$
H_{\mu \nu} \equiv D_{\mu} B_{\nu}-D_{\nu} B_{\mu}+\left\{B_{\mu}, B_{\nu}\right\}+R_{\mu \nu}
$$

It should be emphasized that this consistency condition is clearly satisfied, since it follows from Eqn.(3.1), an equation that has been solved explicitly. Nevertheless the hoped for uniqueness of the string master action $S$ satisfying the master equation $\{S, S\}=0$, implies that a perturbed master action $S+\lambda^{\mu \nu} H_{\mu \nu}$, which would satisfy the master equation to first order in $\lambda$, should just be a field redefined version of the original action. For this to be the case we must demand that there is a hamiltonian $B_{\mu \nu}$ such that

$$
H_{\mu \nu}=\left\{S, B_{\mu \nu}\right\}
$$

If (3.7) holds then (3.5) will follow from the Jacobi identity. More explicitly Eqn.(3.7) reads

$$
D_{\mu} B_{\nu}-D_{\nu} B_{\mu}+\left\{B_{\mu}, B_{\nu}\right\}+R_{\mu \nu}=\left\{S, B_{\mu \nu}\right\}
$$

This is the equation we will analyze. The existence of $B_{\mu \nu}$ amounts to a (partial) cohomology theorem for the string action. It asserts that the $S$-closed hamiltonian $H_{\mu \nu}$ is actually $S$-exact, it equals $\left\{S, B_{\mu \nu}\right\}$. Moreover, it will be seen that the hamiltonian $B_{\mu \nu}$ is defined by moduli spaces of surfaces with two special punctures. 
We can now understand in retrospect that even the existence of the original hamiltonian $B_{\mu}$ of (3.1) amounts to a cohomology theorem. Since we use symplectic connections it follows from $\{S, S\}=0$ that $\left\{S, D_{\mu} S\right\}=0$. Equation (3.1) simply says that the $S$-closed function $D_{\mu} S$ is actually $S$-exact.

\subsection{Preliminary Analysis}

Let us explore the uniqueness of the object $B_{\mu \nu}$ we are after. In equation (3.1) the connection $\Gamma_{\mu}$ is a reference symplectic connection, and can be shifted as long as we preserve the symplectic nature of the connection. Let $\Delta \Gamma_{\mu}^{(i)}$ denote the matrix encoding the change in the connection, and $\Delta \Gamma_{\mu}$ the associated hamiltonian

$$
\Delta \Gamma_{\mu}=-\frac{1}{2}\left\langle\omega_{12}\left|\Delta \Gamma_{\mu}^{(2)}\right| \Psi\right\rangle|\Psi\rangle
$$

We now claim that

$$
\begin{aligned}
\Gamma_{\mu} & \rightarrow \Gamma_{\mu}+\Delta \Gamma_{\mu}, \\
B_{\mu} & \rightarrow B_{\mu}-\Delta \Gamma_{\mu},
\end{aligned}
$$

leaves invariant the background independence condition (3.1). This is a simple consequence of

$$
D_{\mu}(\Gamma+\Delta \Gamma) A=D_{\mu}(\Gamma) A+\{\Delta \Gamma, A\}
$$

which holds for any arbitrary function $A$.

We can now see that the shift (3.10) does not alter the determination of $B_{\mu \nu}$ in Eqn.(3.8). A short computation gives

$$
\begin{aligned}
D_{\mu} B_{\nu}-D_{\nu} B_{\mu}+\left\{B_{\mu}, B_{\nu}\right\} \rightarrow & D_{\mu} B_{\nu}-D_{\nu} B_{\mu}+\left\{B_{\mu}, B_{\nu}\right\} \\
& -D_{\mu} \Delta \Gamma_{\nu}+D_{\nu} \Delta \Gamma_{\mu}-\left\{\Delta \Gamma_{\mu}, \Delta \Gamma_{\nu}\right\},
\end{aligned}
$$

and for the curvature we find

$$
R_{\mu \nu} \rightarrow R_{\mu \nu}+D_{\mu} \Delta \Gamma_{\nu}-D_{\nu} \Delta \Gamma_{\mu}+\left\{\Delta \Gamma_{\mu}, \Delta \Gamma_{\nu}\right\}
$$

It follows immediately that $H_{\mu \nu}$ is left invariant under a shift of the symplectic connection. This proves that the choice of symplectic connection is irrelevant to the computation of $B_{\mu \nu}$. We can therefore use the canonical unit-disk connection $\Gamma$ in the evaluation of $H_{\mu \nu}$ and in the subsequent computation of $B_{\mu \nu}$. 
Let us now consider a different type of transformation. Equation (3.1) is clearly invariant under the shift

$$
B_{\mu} \rightarrow B_{\mu}+\left\{S, \lambda_{\mu}\right\}
$$

Under this shift $H_{\mu \nu}$ transforms as

$$
H_{\mu \nu} \rightarrow H_{\mu \nu}+\left\{S, \mathcal{D}_{\mu} \lambda_{\nu}-\mathcal{D}_{\nu} \lambda_{\mu}\right\}
$$

where we have defined a "gauge covariant" derivative

$$
\mathcal{D}_{\mu} \equiv D_{\mu}+\left\{B_{\mu},\right\}
$$

It follows that under the shift (3.14) our solution for $B_{\mu \nu}$ will shift by

$$
B_{\mu \nu} \rightarrow B_{\mu \nu}+\mathcal{D}_{\mu} \lambda_{\nu}-\mathcal{D}_{\nu} \lambda_{\mu}
$$

This is a true non-uniqueness of $B_{\mu \nu}$. It might be worth remarking that the gauge-covariant derivative introduced above affords some nice notation. Eqn.(3.1) reads $\mathcal{D}_{\mu} S=0$, and $\left[\mathcal{D}_{\mu}, \mathcal{D}_{\nu}\right]=-\left\{H_{\mu \nu}, \quad\right\}$.

\section{Properties of Connections and Covariant Derivatives}

In this section we find the geometrical meaning of the antisymmetric combination $D_{\mu}\left|\widehat{\mathcal{O}}_{\nu}\right\rangle-$ $D_{\nu}\left|\widehat{\mathcal{O}}_{\mu}\right\rangle$. This object will be seen to have a representation in terms of a moduli space $\mathcal{T}_{1}^{2}$ of spheres with three punctures, two of which are special. This moduli space is defined to have real dimension one, but in some sense its natural dimension is two. Since it is a moduli space of three punctured spheres, and the position of three punctures on a sphere define no moduli, the moduli parameters of this moduli space refer to the coordinates at the punctures. Nevertheless it is simpler to think of the punctures as moving, carrying along some canonical coordinates. The derivation of these results will take place in the first three subsections. The last subsection gives a simplified proof of the fact that the connection $\Gamma$ has zero curvature [15]. This is possible due to the geometrical understanding of the antisymmetric combination mentioned above. 


\subsection{Antisymmetric Derivatives}

The purpose of the present subsection is to establish the following result:

$$
D_{\mu}\left|\mathcal{O}_{\nu}\right\rangle-D_{\nu}\left|\mathcal{O}_{\mu}\right\rangle=-\frac{1}{\pi} \int_{|z|<1} \operatorname{dxdy}\left(\mathcal{O}_{\mu}(z, \bar{z})\left|\mathcal{O}_{\nu}\right\rangle-\mathcal{O}_{\nu}(z, \bar{z})\left|\mathcal{O}_{\mu}\right\rangle\right)
$$

This equation indicates that the antisymmetric combination of covariant derivatives using the connection $\Gamma$ is simply related to integrated correlators, and therefore will allow us to understand the antisymmetric combination as arising from a moduli space of surfaces. Since the moving puncture in the right hand side spans a space of real dimension two, the relevant

moduli space can be thought to be of dimension two. Nevertheless, since the rotation $z \rightarrow z e^{i \theta}$ is naturally incorporated in our formalism, it will turn out convenient to think of the moduli space as having one real dimension only. Since the connection $\Gamma$ acts trivially on the ghosts the above equation will imply that

$$
D_{\mu}\left|\widehat{\mathcal{O}}_{\nu}\right\rangle-D_{\nu}\left|\widehat{\mathcal{O}}_{\mu}\right\rangle=-\frac{1}{\pi} \int_{|z|<1} \operatorname{dxdy}\left(\mathcal{O}_{\mu}(z, \bar{z})\left|\widehat{\mathcal{O}}_{\nu}\right\rangle-\mathcal{O}_{\nu}(z, \bar{z})\left|\widehat{\mathcal{O}}_{\mu}\right\rangle\right)
$$

where $|\widehat{\mathcal{O}}\rangle \equiv|c \bar{c} \mathcal{O}\rangle$.

Let us now give a proof of Eqn.(4.1). By definition of the connection coefficients we have

$$
D_{\mu}\left|\mathcal{O}_{\nu}\right\rangle-D_{\nu}\left|\mathcal{O}_{\mu}\right\rangle=\sum_{\gamma_{k}}\left(\Gamma_{\mu \nu}^{k}-\Gamma_{\nu \mu}^{k}\right)\left|\Phi_{k}\right\rangle,
$$

and using (2.15), and (2.16), we find

$$
D_{\mu}\left|\mathcal{O}_{\nu}\right\rangle-D_{\nu}\left|\mathcal{O}_{\mu}\right\rangle=-\frac{1}{\pi} \sum_{\gamma_{k}>2} \frac{H_{\mu \nu}^{k}-H_{\nu \mu}^{k}}{\gamma_{k}-2} \delta_{0, s_{k}}\left|\Phi_{k}\right\rangle .
$$

On the other hand from the definition of operator product, and when integrating over a domain with rotational symmetry:

$$
\mathcal{O}_{\mu}(z, \bar{z}) \mathcal{O}_{\nu}(0)|0\rangle=\frac{1}{2 \pi} \sum_{\gamma_{k}} \frac{H_{\mu \nu}^{k} \delta_{s_{k}, 0}}{r^{4-\gamma_{k}}}\left|\Phi_{k}\right\rangle
$$


We use this equation to form the antisymmetric operator product

$$
\left(\mathcal{O}_{\mu}(z, \bar{z}) \mathcal{O}_{\nu}(0)-\mathcal{O}_{\nu}(z, \bar{z}) \mathcal{O}_{\mu}(0)\right)|0\rangle=\frac{1}{2 \pi} \sum_{\gamma_{k}>2} \frac{\left(H_{\mu \nu}^{k}-H_{\nu \mu}^{k}\right)}{r^{4-\gamma_{k}}} \delta_{s_{k}, 0}\left|\Phi_{k}\right\rangle
$$

In the right hand side we have restricted the sum to $\gamma_{\kappa}>2$ following the discussion of Ref.[ 15] (see below Eqn.(6.23)) where it is shown that the left hand side of (4.6) is integrable over the disk and therefore conformal fields with lower dimension cannot appear. Finally, integrating over the unit disk $|z|<1$ we find

$$
\int_{|z|<1} \operatorname{dxdy}\left(\mathcal{O}_{\mu}(z, \bar{z}) \mathcal{O}_{\nu}(0)-\mathcal{O}_{\nu}(z, \bar{z}) \mathcal{O}_{\mu}(0)\right)|0\rangle=\sum_{\gamma_{k}>2} \frac{\left(H_{\mu \nu}^{k}-H_{\nu \mu}^{k}\right)}{\gamma_{k}-2} \delta_{s_{k}, 0}\left|\Phi_{k}\right\rangle .
$$

Comparing this equation with (4.4) we see that indeed (4.1) has been established. In order to associate a moduli space to the right hand side of (4.1) we now set up some notation to deal with three punctured spheres and local operators.

\subsection{Standard Spheres and Local Operators}

We consider three punctured spheres where the underlying unpunctured sphere is described by uniformizers $z$ and $w$ satisfying $z w=1$. Two of the punctures of the sphere will be considered ordinary; the first, labelled ' 1 ', will be located at $z=0$, with local coordinate $z_{1}=z$, and the second, labelled ' 2 ' will be located at $w=0$, with local coordinate $w_{1}=w$. The third puncture will be thought as special, and will be labelled ' $\overline{1}$ '. Let $S(1)$ denote the sphere

$$
S(1): z_{1}=z, \quad w_{1}=w, \quad w_{\overline{1}}=w-1 .
$$

This sphere is equivalent to the sphere $\mathcal{V}_{3}^{\prime}$ relevant appearing in $(2.21)$, up to the definition of the local coordinate at the special puncture, which does not matter in many cases. Let now $S(t)$ denote a sphere with the special puncture at $w=t$ :

$$
S(t): z_{1}=z, \quad w_{1}=w, \quad w_{\overline{1}}=w-t .
$$

Given the conformal equivalence of all three punctured spheres, $S(t)$ differs from $S(1)$ only by a change of local coordinates at the punctures. In fact, one can readily verify that $S^{\prime}(1)$ 
defined by the following change in the local coordinates of $S(1)$

$$
S^{\prime}(1): \tilde{z}_{1}=z_{1} / t, \quad \tilde{w}_{1}=t w_{1}, \quad \tilde{w}_{\overline{1}}=t w_{\overline{1}},
$$

is conformally equivalent to $S(t)$. All spheres $S(t)$ just differ by scalings of their local coordinates. Nevertheless the parameter $t$ is most easily thought of as specifiying a position. A scaling of the local coordinate on a surface state is realized as $\left\langle\Sigma ; \frac{w_{1}}{t}\right|=\left\langle\Sigma ; w_{1}\right| t^{L_{0}^{(1)}} \bar{t}_{0}^{\bar{L}_{0}^{(1)}}$, and therefore we can relate the sphere surface states as follows

$$
\langle S(t)|=\langle S(1)|\left[t^{L_{0}^{(1)}} \bar{t}^{(1)}\right]\left[t^{-L_{0}^{(2)}} \bar{t}^{-\bar{L}_{0}^{(2)}}\right]\left[t^{L_{0}^{(\overline{1})}} \bar{t}^{(\overline{1})}\right],
$$

where the Fock space label (1) is attached to the puncture at $z=0$, the label (2) is attached to the puncture at $w=0$, and the label $(\overline{1})$ is attached to the special puncture.

The above spheres actually define what we usually mean by local operators. One denotes by $\Phi(t)$ the operator constructed by inserting the state $|\Phi\rangle$ on the special puncture of the sphere $S(t)$, and using a reflector to turn one of the state spaces on the surface state bra into a ket:

$$
2^{\prime}[\Phi(t)]_{2}=\langle S(t) \mid \Phi\rangle_{\overline{1}}\left|R_{12^{\prime}}\right\rangle
$$

Using this definition and (4.11) we derive the operator relations

$$
\begin{aligned}
\Phi(z) & =\frac{1}{z^{h} \bar{z}^{\bar{h}}} z^{L_{0}} \bar{z}^{\bar{L}_{0}} \Phi(1) z^{-L_{0}} \bar{z}^{-\bar{L}_{0}}, \\
\Phi(t z) & =\frac{1}{t^{h} \bar{t}^{\bar{h}}} t^{L_{0}} \bar{t}^{\bar{L}_{0}} \Phi(z) t^{-L_{0}} \bar{t}^{-\bar{L}_{0}} .
\end{aligned}
$$

\subsection{Definition And Properties of $\mathcal{T}_{1}^{2}$}

In this section we will introduce a moduli space $\mathcal{T}_{1}^{2}$ related to $\operatorname{Eqn}(4.1)$. This will be a moduli space of spheres with three punctures, two of which will be special. The real dimension of this space will be one.

We begin by introducing for any $0<u<1$ a space $\tau_{1}^{2}(u)$ of three punctured spheres. The space is of one real dimension and is parametrized by $t$ as follows

$$
\tau_{1}^{2}(u)=\{S(t) \mid t \in[u, 1]\}
$$

where $S(t)$ was defined in (4.9). The surfaces in the moduli space $\tau_{1}^{2}(u)$ are defined to have two special punctures (thus the superscript value) and one ordinary puncture (thus the subscript 
value). The special punctures are the moving puncture which was labelled as $\overline{1}$, and the puncture at $w=0$, which used to be the puncture 2 , but now will be labelled $\overline{2}$. The moving puncture moves from the point $w=u$ up to the point $w=1$. When the moving puncture starts its journey it is closest to the second special puncture.
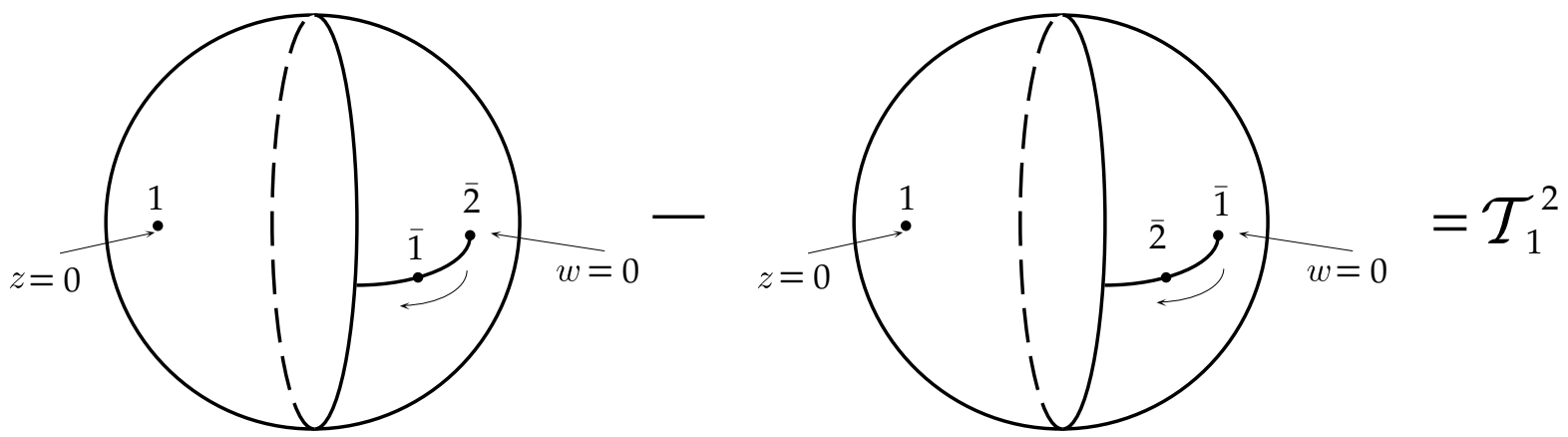

Figure 1 . The family of three punctured spheres that defines the space $\mathcal{T}_{1}^{2}$. This space has two special punctures appearing antisymmetrically. One of the special punctures travels from the point $w=0$, where it coincides with the other special puncture, up to the point $w=1$.

It is useful to consider the limit where we take $u \rightarrow 0$ and the moving puncture starts closer and closer to the second special puncture. The $\operatorname{limit}_{\lim } \tau_{1}^{2}(u)$, however, cannot be expected to be well defined since the operator product expansion of the two marginal states to be inserted at the special punctures will not give an integrable expression (in the parameter $t \in[0,1])$. We must antisymmetrize on the special punctures and define a moduli space $\mathcal{T}_{1}^{2}(u)$ with two special punctures as:

$$
\mathcal{T}_{1}^{2}(u)=\tau_{1}^{2}(u)-P \tau_{1}^{2}(u),
$$

where $P$ simply exchanges the labels $\overline{1}$ and $\overline{2}$ of the special punctures. Moreover, we can now define the limit

$$
\mathcal{T}_{1}^{2} \equiv \lim _{u \rightarrow 0} \mathcal{T}_{1}^{2}(u),
$$

which makes sense because antisymmetrized operator products of marginal operators should be integrable (see Figure 1). It follows from the definition (4.15) that

$$
\partial \mathcal{T}_{1}^{2}(u)=\partial\left(\tau_{1}^{2}(u)-P \tau_{1}^{2}(u)\right)=\left(\mathcal{V}_{3}^{\prime}-P \mathcal{V}_{3}^{\prime}\right)-(S(u)-P S(u)),
$$

where we recall that $\mathcal{V}_{3}^{\prime}=S(1)$ except for the fact that $\mathcal{V}_{3}^{\prime}$ is now considered to have two special 
punctures. Consider now taking now the limit when $u \rightarrow 0$. The antisymmetric combination $(S(u)-P S(u))$ must be taken to vanish since the punctures that are being antisymmetrized are getting close to each other and thus becoming progressively symmetric under their exchange. We then find

$$
\partial \mathcal{T}_{1}^{2}=\mathcal{I} \mathcal{V}_{3}^{\prime}
$$

where anticipating the notation to be discussed in the next section, the operator $\mathcal{I}$ turns an ordinary puncture special, and antisymmetrizes the resulting space in the labels of the special punctures.

We now turn to the derivation of a relation between two dimensional insertions and insertions via $\mathcal{T}_{1}^{2}$. We use the notation

$$
f_{\mathcal{O}_{i} \mathcal{O}_{j}}\left(\tau_{1}^{2}\right) \equiv \int_{\tau_{1}^{2}}\left\langle\Omega_{1 \overline{1} \overline{2}} \mid \mathcal{O}_{i}\right\rangle_{\overline{1}}\left|\mathcal{O}_{j}\right\rangle_{\overline{2}}|\Psi\rangle_{1}
$$

where the canonical CFT-valued moduli space form $\langle\Omega|$ is the usual form used in string field theory (see, for example Ref.[3], Eqn.(3.10)). The states appearing as subscripts in $f$ are inserted on the special punctures, $\mathcal{O}_{i}$ on the punctured labelled $\overline{1}$, and $\mathcal{O}_{j}$ on the puncture labelled $\overline{2}$. We now claim that

Theorem

$$
\left\langle\omega_{12}\left|\frac{1}{\pi} \int_{u<|z|<1} \operatorname{dxdy}\left[\mathcal{O}_{\mu}(z, \bar{z})\right]_{2}\right| \widehat{\mathcal{O}}_{\nu}\right\rangle_{2}|\Psi\rangle_{1}=f_{\mu \nu}\left(\tau_{1}^{2}(u)\right)
$$

where the state space (2) is associated to the unit disk $|z|<1$, and $\widehat{\mathcal{O}}_{\nu}$ is inserted at $z=0$. In the right hand side, the symbol $f_{\mu \nu}$ stands for $f_{\widehat{\mathcal{O}}_{\mu} \widehat{\mathcal{O}}_{\nu}}$.

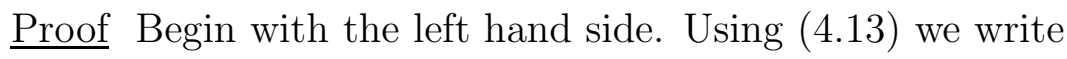

$$
\mathcal{O}_{\mu}(z, \bar{z})=\frac{1}{z \bar{z}} z^{L_{0}} \bar{z}^{\bar{L}_{0}} \mathcal{O}_{\mu}(1) z^{-L_{0}} \bar{z}^{-\bar{L}_{0}}
$$

and using $z=r e^{i \theta}$, the left-hand side becomes,

$$
\text { LHS }=\frac{1}{\pi}(2 \pi)\left\langle\omega_{12}\right|\left(\int_{u}^{1} \operatorname{rdr} \mathrm{r}^{L_{0}+\bar{L}_{0}-2} \int_{0}^{2 \pi} \frac{d \theta}{2 \pi} e^{i \theta\left(L_{0}-\bar{L}_{0}\right)} \mathcal{O}_{\mu}(1) z^{-L_{0}} \bar{z}^{-\bar{L}_{0}}\left|\widehat{\mathcal{O}}_{\nu}\right\rangle\right)_{2}|\Psi\rangle_{1} .
$$


Since $L_{0}\left|\mathcal{O}_{\nu}\right\rangle=\bar{L}_{0}\left|\mathcal{O}_{\nu}\right\rangle=0$, and $\left\langle\omega_{12}\right|\left(L_{0}-\bar{L}_{0}\right)_{2}=0$, we find

$$
\operatorname{LHS}=2\left\langle\omega_{12}\right|\left(\int_{u}^{1} \mathrm{dr} \mathrm{r}^{L_{0}+\bar{L}_{0}-1} \mathcal{O}_{\mu}(1)\left|\widehat{\mathcal{O}}_{\nu}\right\rangle\right)_{2}|\Psi\rangle_{1}
$$

Now consider the right hand side (RHS), which by definition $((4.19))$ is the integral of the canonical string one-form over the space $\tau_{1}^{2}(u)$

$$
\operatorname{RHS}=\int_{\tau_{1}^{2}(u)}\left\langle\Omega_{1 \overline{1} \overline{2}}^{[1]} \mid \widehat{\mathcal{O}}_{\mu}\right\rangle_{\overline{1}}\left|\widehat{\mathcal{O}}_{\nu}\right\rangle_{\overline{2}}|\Psi\rangle_{1}
$$

Since $\tau_{1}^{2}(u)$ is a moduli space of three punctured spheres the canonical normalization factor of the string form equals one, and the string form is simply the surface state with the appropriate antighost insertions. We have (using $z=t+w=h_{t}(w)$ in the setup of Ref.[21], Eqns.(2.30$33)$ )

$$
\begin{aligned}
\left\langle\Omega_{1 \overline{1} \overline{2}}^{[1]} \mid \widehat{\mathcal{O}}_{\mu}\right\rangle_{\overline{1}} & =\left\langle S(t)_{1 \overline{1} \overline{2}}\left|d t \mathbf{b}\left(\frac{\partial}{\partial t}\right)\right| \widehat{\mathcal{O}}_{\mu}\right\rangle_{\overline{1}} \\
& =-d t\left\langle S(t)_{1 \overline{1} \overline{2}}\right|\left(\left(b_{-1}+\bar{b}_{-1}\right) c_{1} \bar{c}_{1}\left|\widehat{\mathcal{O}}_{\mu}\right\rangle\right)_{\overline{1}} \\
& =d t\left\langle S(t)_{1 \overline{1} \overline{2}}\right|\left(\left(c_{1}-\bar{c}_{1}\right)\left|\mathcal{O}_{\mu}\right\rangle\right)_{\overline{1}}, \\
& =d t\left\langle R_{1 \overline{2}}\right|\left(c \mathcal{O}_{\mu}(t)-\bar{c} \mathcal{O}_{\mu}(t)\right)_{\overline{2}} .
\end{aligned}
$$

Now we use $(t=z=\bar{z})$

$$
\left\{\begin{array}{l}
c \mathcal{O}_{\mu}(t) \\
\bar{c} \mathcal{O}_{\mu}(t)
\end{array}\right\}=t^{L_{0}+\bar{L}_{0}-1}\left\{\begin{array}{l}
c \mathcal{O}_{\mu}(1) \\
\bar{c} \mathcal{O}_{\mu}(1)
\end{array}\right\} t^{-L_{0}-\bar{L}_{0}}
$$

to find

$$
\operatorname{RHS}=\left\langle R_{1 \overline{2}}\right|\left(\int_{u}^{1} d t t^{L_{0}+\bar{L}_{0}-1}(c(1)-\bar{c}(1)) \mathcal{O}_{\mu}(1)\left|\widehat{\mathcal{O}}_{\nu}\right\rangle\right)_{\overline{2}}|\Psi\rangle_{1}
$$

Making use of $|\Psi\rangle=b_{0}^{-} c_{0}^{-}|\Psi\rangle$, the reflection property $\left\langle R_{1 \overline{2}}\right| b_{0}^{-(\overline{2})}=\left\langle R_{1 \overline{2}}\right| b_{0}^{-(1)}$, and the anti- 
commutator $\left\{b_{0}^{-}, c(1)-\bar{c}(1)\right\}=2$, we find that the right hand side becomes

$$
\begin{aligned}
\operatorname{RHS} & =-2\left\langle R_{1 \overline{2}}^{\prime}\right|\left(\int_{u}^{1} d t t^{L_{0}+\bar{L}_{0}-1} \mathcal{O}_{\mu}(1)\left|\widehat{\mathcal{O}}_{\nu}\right\rangle\right)_{\overline{2}} c_{0}^{-}|\Psi\rangle_{1} \\
& =2\left\langle\omega_{1 \overline{2}}\right|\left(\int_{u}^{1} d t t^{L_{0}+\bar{L}_{0}-1} \mathcal{O}_{\mu}(1)\left|\widehat{\mathcal{O}}_{\nu}\right\rangle\right)_{\overline{2}}|\Psi\rangle_{1}
\end{aligned}
$$

Comparison with (4.23) establishes the desired result (eqn.(4.20)).

Given the definition

$$
U_{\mathcal{O}}^{(2)}=\left\langle\omega_{12} \mid \mathcal{O}\right\rangle_{1}\left|\Psi_{2}\right\rangle
$$

we have shown that

$$
U_{-\frac{1}{\pi} \int_{u<|z|<1}^{(2)} \operatorname{dxdy}\left[\mathcal{O}_{\mu}(z, \bar{z})\right]\left|\widehat{\mathcal{O}}_{\nu}\right\rangle}=f_{\mu \nu}\left(\tau_{1}^{2}(u)\right)
$$

Combining this result with (4.2) we now write

$$
U_{D_{\mu}\left|\widehat{\mathcal{O}}_{\nu}\right\rangle-D_{\nu}\left|\widehat{\mathcal{O}}_{\mu}\right\rangle}^{(2)}=f_{\mu \nu}\left(\mathcal{T}_{1}^{2}\right)
$$

where we made use of (4.15) and have taken the limit $u \rightarrow 0$ which is allowed on the antisymmetric combination. This last equation expresses the fact that an insertion of the state $D_{\mu}\left|\widehat{\mathcal{O}}_{\nu}\right\rangle-D_{\nu}\left|\widehat{\mathcal{O}}_{\mu}\right\rangle$ can be achieved via the insertion of the states $\left|\widehat{\mathcal{O}}_{\mu}\right\rangle$ and $\left|\widehat{\mathcal{O}}_{\nu}\right\rangle$ on the moduli space $\mathcal{T}_{1}^{2}$ of three punctured spheres.

\subsection{Simplifying A CURVATURE COMPUtATion}

It is now possible to use the results of the previous subsections to simplify the proof that the connection $\Gamma$ has zero curvature [15]. Recall the definition (2.14), which for convenience we reproduce here

$$
D_{\mu}\langle\Sigma|=-\frac{1}{\pi} \int_{p \in \widehat{\Sigma}} d \mu(p)\left\langle\Sigma ; p \mid \mathcal{O}_{\mu}(p)\right\rangle,
$$

In order to be able to take a further covariant derivative we must specify the coordinate disk to be used for the point $p$ as it moves around in $\widehat{\Sigma}$. Let $\Delta_{p}$ denote this disk, which we fix in such a way that for any puncture $P_{i}$, as the point $p$ approaches the boundary of the disk $D_{i}$ 
the disk $\Delta_{p}$ does not contain the puncture $P_{i}$. We can then take another derivative to find, using the convenient notation introduced in [22]

$$
\begin{aligned}
D_{\nu} D_{\mu}\langle\Sigma|= & -\frac{1}{\pi} \int_{p \in \widehat{\Sigma}} d \mu(p)\langle\Sigma ; p|\left(D_{\nu}\left|\mathcal{O}_{\mu}(p)\right\rangle\right) \\
& +\frac{1}{\pi^{2}} \int_{p \in \widehat{\Sigma}} d \mu(p) \int_{\substack{q \in \widehat{\Sigma} \\
q \notin \Delta_{p}}} d \mu(q)\left\langle\Sigma ; p, q \mid \mathcal{O}_{\mu}(p)\right\rangle\left|\mathcal{O}_{\nu}(q)\right\rangle \\
& -\frac{1}{\pi^{2}} \sum_{i} \int_{p \in \widehat{\Sigma}} d \mu(p) \int_{q \in D_{i} \cap \Delta_{p}} d \mu(q)\left\langle\Sigma ; p, q \mid \mathcal{O}_{\mu}(p)\right\rangle\left|\mathcal{O}_{\nu}(q)\right\rangle
\end{aligned}
$$

The first term in the right hand side is simply computing the covariant derivative of the state $\left|\mathcal{O}_{\mu}\right\rangle$, while the second and third terms deal with the covariant derivative of $\langle\Sigma ; p|$. When the disk $\Delta_{p}$ lies completely within $\widehat{\Sigma}$ the third term vanishes and the second term computes the correct derivative. When the disk $\Delta_{p}$ intersects a disk $D_{i}$, the intersection $D_{i} \cap \Delta_{p}$ must be counted negatively, and the third term does this. This is readily understood: the definition of $\Gamma$ requires integrating over the surface minus all the coordinate disks $\Sigma-\cup D_{i}-\Delta_{p}=\widehat{\Sigma}-\Delta_{p}$. This region, however, can be written as $\left[\widehat{\Sigma}-\widehat{\Sigma} \cap \Delta_{p}\right]-\left[\Delta_{p}-\widehat{\Sigma} \cap \Delta_{p}\right]$. The first term in this difference is taken care by the second term in the above right hand side, while the second term represents the integral over $\Delta_{p} \cap D_{i}$ and counts negatively, as the third term in the above right hand side does. This last term actually gives rise to a divergence when the point $p$ approaches the boundary of the disks $D_{i}$. This divergence will cancel out in the commutator which we now compute

$$
\begin{aligned}
{\left[D_{\nu}, D_{\mu}\right]\langle\Sigma|=} & -\frac{1}{\pi} \int_{p \in \widehat{\Sigma}} d \mu(p)\left\langle\Sigma ; p\left|\left(D_{\nu}\left|\mathcal{O}_{\mu}(p)\right\rangle-D_{\mu}\left|\mathcal{O}_{\nu}(p)\right\rangle\right)\right.\right. \\
& +\frac{1}{\pi^{2}} \int_{p \in \widehat{\Sigma}} d \mu(p) \int_{\substack{q \in \widehat{\Sigma} \\
q \notin \Delta_{p}}} d \mu(q)\left\langle\Sigma ; p, q\left|\left(\left|\mathcal{O}_{\mu}(p)\right\rangle\left|\mathcal{O}_{\nu}(q)\right\rangle-\left|\mathcal{O}_{\nu}(p)\right\rangle\left|\mathcal{O}_{\mu}(q)\right\rangle\right)\right.\right. \\
& -\frac{1}{\pi^{2}} \sum_{i} \int_{p \in \widehat{\Sigma}} d \mu(p) \int_{q \in D_{i} \cap \Delta_{p}} d \mu(q)\left\langle\Sigma ; p, q\left|\left(\left|\mathcal{O}_{\mu}(p)\right\rangle\left|\mathcal{O}_{\nu}(q)\right\rangle-\left|\mathcal{O}_{\nu}(p)\right\rangle\left|\mathcal{O}_{\mu}(q)\right\rangle\right)\right.\right.
\end{aligned}
$$




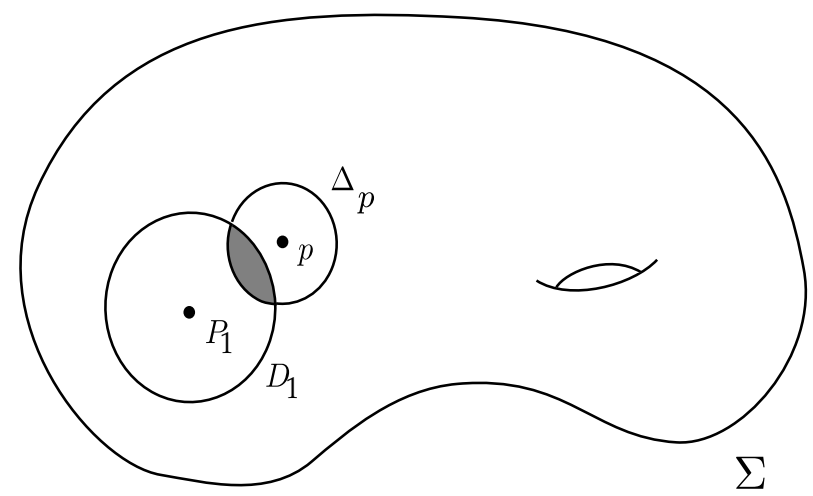

Figure 2. This figure refers to the computation of curvature for the connection $\Gamma$. We see the first moving puncture at $p$ and its disk $\Delta_{p}$ which happens to intersect the unit disk $D_{1}$ of the ordinary puncture $P_{1}$. In this case the shaded region $\Delta_{p} \cap D_{1}$ is a region of integration for the second puncture and contributes with a negative sign.

Using (4.1) the first line in the above equation can now be written as

$$
+\frac{1}{\pi^{2}} \int_{p \in \widehat{\Sigma}} d \mu(p) \int_{q \in \Delta_{p}} d \mu(q)\left\langle\Sigma ; p, q\left|\left(\left|\mathcal{O}_{\mu}(p)\right\rangle\left|\mathcal{O}_{\nu}(q)\right\rangle-\left|\mathcal{O}_{\nu}(p)\right\rangle\left|\mathcal{O}_{\mu}(q)\right\rangle\right) .\right.\right.
$$

Back in (4.34) we see that the six terms combine naturally into two groups, each with three terms, one having the states $\left|\mathcal{O}_{\mu}(p)\right\rangle\left|\mathcal{O}_{\nu}(q)\right\rangle$ in the integrand, and the other having the states $\left|\mathcal{O}_{\nu}(p)\right\rangle\left|\mathcal{O}_{\mu}(q)\right\rangle$ in the integrand. The first group can be written as

$$
\frac{1}{\pi^{2}} \int_{p \in \widehat{\Sigma}} d \mu(p)\left[\int_{q \in \Delta_{p}} d \mu(q)+\int_{\substack{q \in \widehat{\Sigma} \\ q \notin \Delta_{p}}} d \mu(q)-\sum_{i} \int_{q \in D_{i} \cap \Delta_{p}} d \mu(q)\right]\left\langle\Sigma ; p, q \mid \mathcal{O}_{\mu}(p)\right\rangle\left|\mathcal{O}_{\nu}(q)\right\rangle .
$$

There are two classes of points $p$. In the first class $\Delta_{p}$ is completely within $\widehat{\Sigma}$, the last integral in the bracket vanishes and the first two build the integral of $q$ over the complete $\widehat{\Sigma}$. In the second class $\Delta_{p}$ intersects a disk $D_{i}$. In this case the first and last integral within the bracket build the integral over the part of $\Delta_{p}$ in $\widehat{\Sigma}$, and together with the second integral they again give the integral of $q$ over the complete surface $\widehat{\Sigma}$. Thus in both cases the integral over $q$ extends over $\widehat{\Sigma}$ and we get

$$
\frac{1}{\pi^{2}} \int_{p \in \widehat{\Sigma}} d \mu(p) \int_{q \in \widehat{\Sigma}} d \mu(q)\left\langle\Sigma ; p, q \mid \mathcal{O}_{\mu}(p)\right\rangle\left|\mathcal{O}_{\nu}(q)\right\rangle
$$

Since the integrations do not distinguish between $p$ and $q$, the above object is symmetric under the exchange of $\mu$ and $\nu$. This being the case it cancels against the other group of three terms 
which gives an identical contribution except for the exchange of $\mu$ and $\nu$. This shows that the the right hand side of (4.34) vanishes, and therefore, that the connection $\Gamma$ has zero curvature. This proof will motivate our definition of the operator $\mathcal{K}$ in the next section, and the zero curvature property will be related to $\mathcal{K}^{2}=0$.

\section{BV algebra on Riemann Surfaces with Special Punctures}

The purpose of the present section is to extend the definition of the BV algebra of moduli spaces of punctured Riemann surfaces [3,1] to the case when the surfaces have special punctures. We will define a new operator $\mathcal{I}$ and the earlier definition of $\mathcal{K}$ will have to be extended. Some useful properties of these operators will be derived.

For clarity we will denote as $\mathcal{A}$ spaces moduli spaces whose surfaces have no special punctures. Moduli spaces whose surfaces have a number of special punctures will be denoted as $\mathcal{B}$ spaces. An $\mathcal{A}$ space is a particular case of a $\mathcal{B}$ space with zero special punctures. $\mathcal{B}$ spaces will be taken to be symmetric under the exchange of labels of any pair of ordinary punctures and antisymmetric under the exchange of labels of any pair of special punctures. The special punctures are not used for sewing, but the antisymmetry property must be preserved under any operation. A $\mathcal{B}$ space will be said to be of type $(n, \bar{n})$, and sometimes to be written as $\mathcal{B}_{n}^{\bar{n}}$ when it has $n$ ordinary punctures and $\bar{n}$ special punctures (the bar in $\bar{n}$ is not meant to imply any relation to $n)$. In a type $(n, \bar{n})$ space the ordinary punctures are labelled from 1 to $n$, and the special punctures are labelled from $\overline{1}$ to $\bar{n}$. The ordering of the ordinary punctures is irrelevant, but the ordering of the special punctures is important for signs to work out.

A useful notion for a $\mathcal{B}$ space is that of a primitive domain. A primitive domain $\overline{\mathcal{B}}$ of a $\mathcal{B}$ space with $\bar{n}$ special punctures is a subspace of $\mathcal{B}$ such that $\mathcal{B}$ is reproduced by the disjoint union of $\overline{\mathcal{B}}$ with the copies obtained by antisymmetrizing in all the special punctures, a total of $n$ ! properly signed copies of $\overline{\mathcal{B}}$. Roughly speaking one cannot find in the primitive domain two surfaces that just differ by the exchange of two special punctures, such surfaces may appear in the boundary of the primitive domain. The primitive domain of a $\mathcal{B}$ space is not unique. 


\subsection{EXTENDED MODULI SPACES AND THE ANTIBRACKET}

Let us first recall the definition of the antibracket for ordinary moduli spaces of punctured surfaces. Given two moduli spaces $\mathcal{A}_{1}$ and $\mathcal{A}_{2}$ their antibracket $\left\{\mathcal{A}_{1}, \mathcal{A}_{2}\right\}$ is defined as follows: pick any particular labeled puncture out of the $n_{1}$ punctures of $\mathcal{A}_{1}$ and any particular labeled puncture out of the $n_{2}$ punctures in $\mathcal{A}_{2}$ and do twist sewing. In the resulting space of surfaces, having a total of $n=\left(n_{1}-1\right)+\left(n_{2}-1\right)$ punctures we add over all inequivalent ways of splitting the $n$ punctures, now labelled from 1 to $n$, into two groups of unordered punctures, one with $\left(n_{1}-1\right)$ punctures and the other one with $\left(n_{2}-1\right)$ punctures. With this definition one readily verifies that [3]

$$
\begin{gathered}
\left\{\mathcal{A}_{1}, \mathcal{A}_{2}\right\}=-(-)^{\left(\mathcal{A}_{1}+1\right)\left(\mathcal{A}_{2}+1\right)}\left\{\mathcal{A}_{2}, \mathcal{A}_{1}\right\}, \\
(-)^{\left(A_{1}+1\right)\left(A_{3}+1\right)}\left\{\left\{\mathcal{A}_{1}, \mathcal{A}_{2}\right\}, \mathcal{A}_{3}\right\}+\text { cyclic }=0, \\
\partial\left\{\mathcal{A}_{1}, \mathcal{A}_{2}\right\}=\left\{\partial \mathcal{A}_{1}, \mathcal{A}_{2}\right\}+(-)^{\mathcal{A}_{1}+1}\left\{\mathcal{A}_{1}, \partial \mathcal{A}_{2}\right\} .
\end{gathered}
$$

Let us now define the antibracket $\left\{\mathcal{B}_{1}, \mathcal{B}_{2}\right\}^{\prime}$ of two $\mathcal{B}$ spaces. We include a prime because the final antibracket we are interested in will have an extra sign factor. We take $\mathcal{B}_{1}$ to be an $\left(n_{1}, \bar{n}_{1}\right)$ space and $\mathcal{B}_{2}$ to be an $\left(n_{2}, \bar{n}_{2}\right)$ space. We first compute the antibracket as if there were only ordinary punctures. In all of the resulting surfaces the total number of special punctures is now $\bar{n}_{1}+\bar{n}_{2}$. The labels of the special punctures that arise from $\mathcal{B}_{1}$ are left unchanged, and the punctures that arise from $\mathcal{B}_{2}$ are consecutively labelled $\bar{n}_{1}+1, \cdots, \bar{n}_{1}+\bar{n}_{2}$. Take the complete set of labelled special punctures and consider the inequivalent splittings of them into two groups $\left(i_{1} \cdots i_{\bar{n}_{1}}\right)$ and $\left(i_{1} \cdots i_{\bar{n}_{2}}\right)$ with $\bar{n}_{1}$ and $\bar{n}_{2}$ elements respectively. Two splitting are equivalent if the resulting groups are seen to contain the same labels, regardless of the order. For each splitting label the ordered punctures in the $\mathcal{B}_{1}$ side of the sewn surface using the labels $\left(i_{1} \cdots i_{\bar{n}_{1}}\right)$ and label the ordered punctures in the $\mathcal{B}_{2}$ side of the sewn surface using the labels $\left(i_{1} \cdots i_{\bar{n}_{2}}\right)$. The sign factor assigned to this splitting is the sign of the permutation necessary to bring $\left(i_{1}, \cdots, i_{\bar{n}_{1}}, i_{1}, \cdots, i_{\bar{n}_{2}}\right)$ to standard ascending order. Add over all inequivalent splittings and that is the desired antibracket. It follows from this definition that this antibracket will have the exchange property

$$
\left\{\mathcal{B}_{1}, \mathcal{B}_{2}\right\}^{\prime}=-(-)^{\left(\mathcal{B}_{1}+1\right)\left(\mathcal{B}_{2}+1\right)+\bar{n}_{1} \bar{n}_{2}}\left\{\mathcal{B}_{2}, \mathcal{B}_{1}\right\}^{\prime}
$$

where the first part of the sign factor comes from the standard antibracket, and the $\bar{n}_{1} \bar{n}_{2}$ part arises because in the exchanged antibracket the special punctures of $\mathcal{B}_{2}$ should be labelled first. 
The derivative property of the antibracket is not changed

$$
\partial\left\{\mathcal{B}_{1}, \mathcal{B}_{2}\right\}^{\prime}=\left\{\partial \mathcal{B}_{1}, \mathcal{B}_{2}\right\}^{\prime}+(-)^{\mathcal{B}_{1}+1}\left\{\mathcal{B}_{1}, \partial \mathcal{B}_{2}\right\}^{\prime} .
$$

Finally, the Jacobi identity now takes the form

$$
(-)^{\left(\mathcal{B}_{1}+1\right)\left(\mathcal{B}_{3}+1\right)+\bar{n}_{1} \bar{n}_{3}}\left\{\left\{\mathcal{B}_{1}, B_{2}\right\}^{\prime}, \mathcal{B}_{3}\right\}^{\prime}+\text { Cyclic }(1 \rightarrow 2 \rightarrow 3)=0,
$$

where the extra sign factors with respect to the standard relation (2.6) appear due to the special punctures.

It is useful now to introduce the final version $\{$,$\} of the antibracket of two \mathcal{B}$ spaces by including a sign factor that will make the identities take a more familiar form. We let

$$
\left\{\mathcal{B}_{1}, \mathcal{B}_{2}\right\} \equiv(-)^{\bar{n}_{1}\left(1+\mathcal{B}_{2}\right)}\left\{\mathcal{B}_{1}, \mathcal{B}_{2}\right\}^{\prime}
$$

With this redefinition the last three identities will read

$$
\begin{gathered}
\left\{\mathcal{B}_{1}, \mathcal{B}_{2}\right\}=-(-)^{\left(\mathcal{B}_{1}+\bar{n}_{1}+1\right)\left(\mathcal{B}_{2}+\bar{n}_{2}+1\right)}\left\{\mathcal{B}_{2}, \mathcal{B}_{1}\right\}, \\
\partial\left\{\mathcal{B}_{1}, \mathcal{B}_{2}\right\}=\left\{\partial \mathcal{B}_{1}, \mathcal{B}_{2}\right\}+(-)^{\mathcal{B}_{1}+\bar{n}_{1}+1}\left\{\mathcal{B}_{1}, \partial \mathcal{B}_{2}\right\} \\
(-)^{\left(\mathcal{B}_{1}+\bar{n}_{1}+1\right)\left(\mathcal{B}_{3}+\bar{n}_{3}+1\right)}\left\{\left\{\mathcal{B}_{1}, B_{2}\right\}, \mathcal{B}_{3}\right\}+\operatorname{Cyclic}(1 \rightarrow 2 \rightarrow 3)=0,
\end{gathered}
$$

where we see that we have the standard identities if we assign to a $\mathcal{B}$ space a grassmanality equal to the real dimension of the space plus the number of special punctures.

\subsection{THE $\mathcal{K}$ OPERATOR}

On a surface that has only ordinary punctures the operator $\mathcal{K}$ has been defined earlier [ 3]; it inserts a special puncture, labelled $\overline{1}$ throughout the surface minus the unit disks around the ordinary punctures. In such cases we have

$$
\begin{aligned}
\mathcal{K}\left(\left\{\mathcal{A}_{1}, \mathcal{A}_{2}\right\}\right) & =\left\{\mathcal{K} \mathcal{A}_{1}, \mathcal{A}_{2}\right\}+\left\{\mathcal{A}_{1}, \mathcal{K} \mathcal{A}_{2}\right\} \\
{[\partial, \mathcal{K}] } & =-\left\{\mathcal{V}_{3}^{\prime},\right\} .
\end{aligned}
$$

Now consider the case when we act on a surface which has one special puncture, labelled $\overline{1}$, in addition to several ordinary punctures. We will define the operation $\mathcal{K}$ to add a new special 
puncture, labelled $\overline{2}$ and to insert it throughout the surface minus unit disks of the ordinary punctures, just ignoring the special puncture $\overline{1}$. To this result one must subtract the same surfaces with the labels $\overline{1}$ and $\overline{2}$ exchanged, thus producing a space of surfaces with two special punctures satisfying the requisite antisymmetry property (see Figure 3). The special punctures collide but divergences will be avoided by antisymmetrization on the special punctures. If the original surface has $\bar{n}$ special punctures $\mathcal{K}$ will insert a puncture labelled $(\bar{n}+1)$ throughout the surface minus the unit disks around the ordinary punctures. It will then subtract $\bar{n}$ copies of this surface where the label of the new special puncture is exchanged with each of the labels of the original special punctures, one at a time. This defines $\mathcal{K B}$. At the level of primitive domains we have that $\overline{\mathcal{K B}}=\mathcal{K} \overline{\mathcal{B}}$, where in the right hand side the $\mathcal{K}$ simply adds the $(\bar{n}+1)$ puncture.

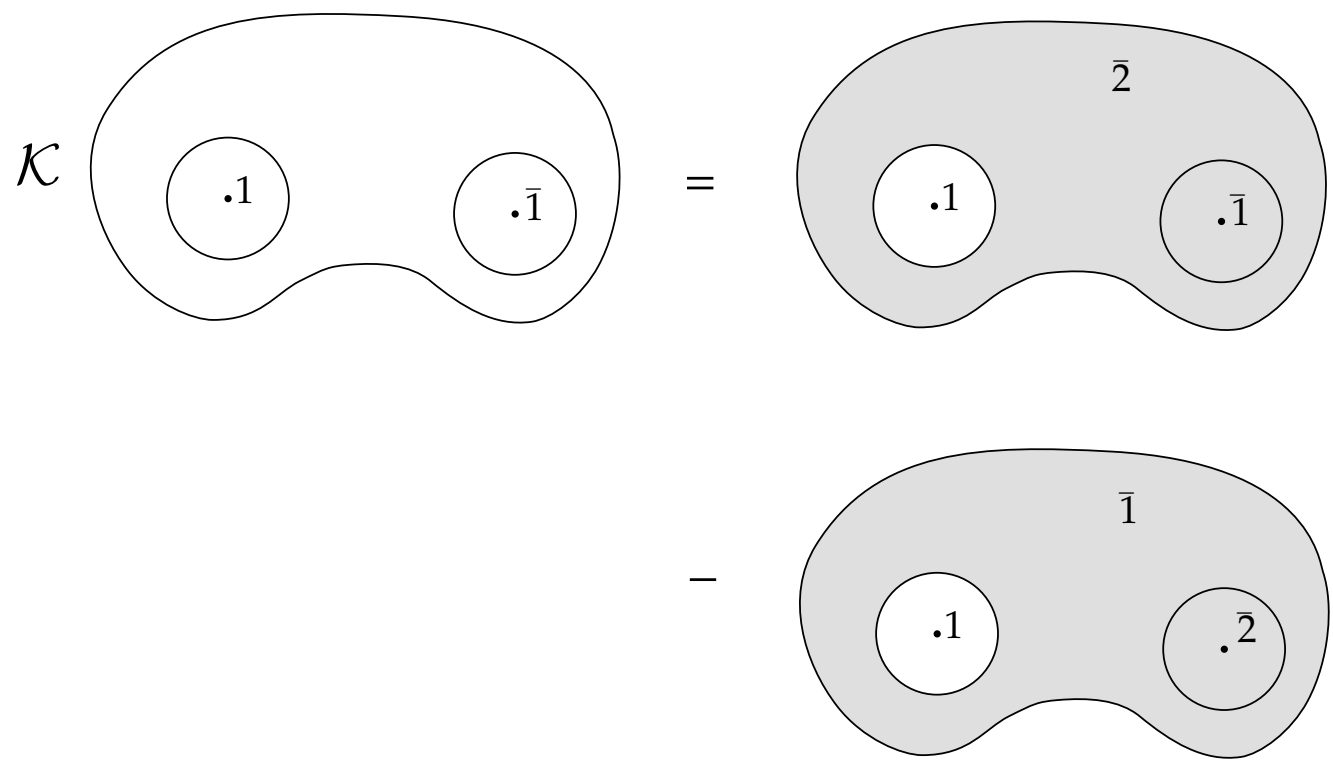

Figure 3. This figure illustrates the definition of the operator $\mathcal{K}$ when it acts on a surface with one ordinary puncture 1 and one special puncture $\overline{1}$. The second special puncture $\overline{2}$ is added throughout the surface minus the unit disk around the ordinary puncture. Then one antisymmetrizes on the special punctures.

We now claim that the following three equations hold

$$
\begin{aligned}
\mathcal{K}\left(\left\{\mathcal{B}^{\bar{n}_{1}}, \mathcal{B}^{\bar{n}_{2}}\right\}^{\prime}\right) & =(-)^{\bar{n}_{2}}\left\{\mathcal{K} \mathcal{B}^{\bar{n}_{1}}, \mathcal{B}^{\bar{n}_{2}}\right\}^{\prime}+\left\{\mathcal{B}^{\bar{n}_{1}}, \mathcal{K} \mathcal{B}^{\bar{n}_{2}}\right\}^{\prime} \\
{[\partial, \mathcal{K}] \mathcal{B}^{\bar{n}} } & =-(-)^{\bar{n}}\left\{\mathcal{V}_{3}^{\prime}, \mathcal{B}^{\bar{n}}\right\}^{\prime}=-(-)^{\mathcal{B}}\left\{\mathcal{B}^{\bar{n}}, \mathcal{V}_{3}^{\prime}\right\}^{\prime} \\
\mathcal{K} \mathcal{K} & =0 .
\end{aligned}
$$

Let us begin with the last equation. It holds acting on a space with no special punctures because 
the two special punctures end up integrated over the same region, and the antisymmetry condition will make them cancel out. This is the way our computation of curvature in the last section worked out. On a space that already has some special punctures the regions of integration still agree (given that all special punctures are ignored when inserting the new puncture) and the configurations where the two new special punctures have the labels $\bar{n}+1$ and $\bar{n}+2$ clearly cancel. All other configurations will also cancel out in pairs.

The first equation is also simple to understand. Consider the configuration where in the left hand side $\mathcal{K}$ inserts the puncture labelled $\left(\bar{n}_{1}+\bar{n}_{2}+1\right)$ on the sewn surface. If the puncture lies on the $\mathcal{B}^{\bar{n}_{2}}$ surface then the labelling of the punctures is consistent with the antibracket of the second term in the right hand side and no sign factor is required. If it lies on the $\mathcal{B}^{\bar{n}_{1}}$ surface, to compare with the labelling implied by the first term in the right hand side we have to relabel all the punctures in the second surface, a total of $\bar{n}_{2}$ exchanges giving rise to the sign factor.

In the second equation the sign factor that appears in addition to the sign factor in (5.11) also arises for similar reasons. In the left hand side, the puncture that ends on the boundary of a disk is the $\bar{n}+1$ puncture. On the other hand, in the right hand side it is the first special puncture that ends there, since $\mathcal{V}_{3}^{\prime}$ appears first in the antibracket. The rearrangement of labels requires $\bar{n}$ exchanges, thus the sign factor.

Finally, using the unprimed antibracket $((5.7))$ the equations listed in (5.12) become

$$
\begin{aligned}
\mathcal{K}\left(\left\{\mathcal{B}^{\bar{n}_{1}}, \mathcal{B}^{\bar{n}_{2}}\right\}\right) & =(-)^{\mathcal{B}_{2}+\bar{n}_{2}+1}\left\{\mathcal{K} \mathcal{B}^{\bar{n}_{1}}, \mathcal{B}^{\bar{n}_{2}}\right\}+\left\{\mathcal{B}^{\bar{n}_{1}}, \mathcal{K} \mathcal{B}^{\bar{n}_{2}}\right\} \\
{[\partial, \mathcal{K}] \mathcal{B}^{\bar{n}} } & =(-)^{\mathcal{B}+\bar{n}}\left\{\mathcal{V}_{3}^{\prime}, \mathcal{B}^{\bar{n}}\right\} \\
\mathcal{K} \mathcal{K} & =0
\end{aligned}
$$

\section{3. $\mathcal{I}$ OPERATOR AND $\{\mathcal{I}, \mathcal{K}\}$.}

The operator $\mathcal{I}$ acting on a moduli space whose surfaces have only ordinary punctures, will pick a fixed label puncture and convert it into a special puncture, labelled $\overline{1}$. Which puncture is chosen is irrelevant since the space is symmetric under any exchange of ordinary punctures (the remaining ordinary punctures may be relabelled with consecutive labels, if necessary). This operation was not defined explicitly earlier, underbars were used to indicate spaces of ordinary punctures that happened to get a special state inserted in one of the punctures. It 
is useful, however, to bring this operation into the open. The following identities are simple consequences of the above definition

$$
\begin{aligned}
\mathcal{I}\left(\left\{\mathcal{A}_{1}, \mathcal{A}_{2}\right\}\right) & =\left\{\mathcal{I} \mathcal{A}_{1}, \mathcal{A}_{2}\right\}+\left\{\mathcal{A}_{1}, \mathcal{I} \mathcal{A}_{2}\right\} \\
{[\partial, \mathcal{I}] } & =0
\end{aligned}
$$

On a $\mathcal{B}$ space with $\bar{n}$ special punctures the operator $\mathcal{I}$ will change one of the ordinary punctures into a new special puncture and will label it $\bar{n}+1$. Then it will subtract $\bar{n}$ copies, exchanging the label of the new special puncture with each of the $\bar{n}$ labels of the original special punctures (see Figure 4). This defines $\mathcal{I B}$. At the level of fundamental domains we have $\overline{\mathcal{I} \mathcal{B}}=\mathcal{I} \overline{\mathcal{B}}$, where the $\mathcal{I}$ in the right hand side just converts one puncture and labels it $(\bar{n}+1)$. The operator $\mathcal{I}$ is rather similar to $\mathcal{K}$, except that the dimensionality of the space of surfaces is not changed by the action of $\mathcal{I}$, nor are new collisions of punctures induced. We claim that the following identities hold

$$
\begin{aligned}
\mathcal{I}\left\{\mathcal{B}^{\bar{n}_{1}}, \mathcal{B}^{\bar{n}_{2}}\right\} & =(-)^{\mathcal{B}_{2}+\bar{n}_{2}+1}\left\{\mathcal{I B}^{\bar{n}_{1}}, \mathcal{B}^{\bar{n}_{2}}\right\}+\left\{\mathcal{B}^{\bar{n}_{1}}, \mathcal{I B}^{\bar{n}_{2}}\right\} \\
{[\partial, \mathcal{I}] } & =0 \\
\mathcal{I} \mathcal{I} & =0
\end{aligned}
$$

The first equation here follows directly from the first equation of (5.14) with the sign factors identical to those appearing in (5.13). The second identity needs no explanation. The last identity follows because after acting twice with $\mathcal{I}$, a space will have two ordinary punctures turned special, and the antisymmetrization on the special punctures will give zero due to the original symmetry under exchange of ordinary punctures.

There is one important relation we discuss now. We claim that

$$
\mathcal{K} \mathcal{I}+\mathcal{I} \mathcal{K}=\left\{, \mathcal{T}_{1}^{2}\right\}
$$

where $\mathcal{T}_{1}^{2}$ is the $\mathcal{B}$ space with two special punctures introduced in section 4.3.

As a preliminary point note that $\left\{, \mathcal{T}_{1}^{2}\right\}=\left\{, \mathcal{T}_{1}^{2}\right\}^{\prime}$, regardless of the moduli space appearing in the first slot of the antibracket. This follows from Eqn.(5.7) given that the dimension of $\mathcal{T}_{1}^{2}$ is one. We can therefore discuss (5.16) using the primed antibracket.

As usual it is convenient to first discuss this equation acting on ordinary moduli spaces. It is in fact sufficient to discuss the case when we act on a single surface having a single ordinary puncture and an associated canonical disk. This is illustrated in Fig.5. 


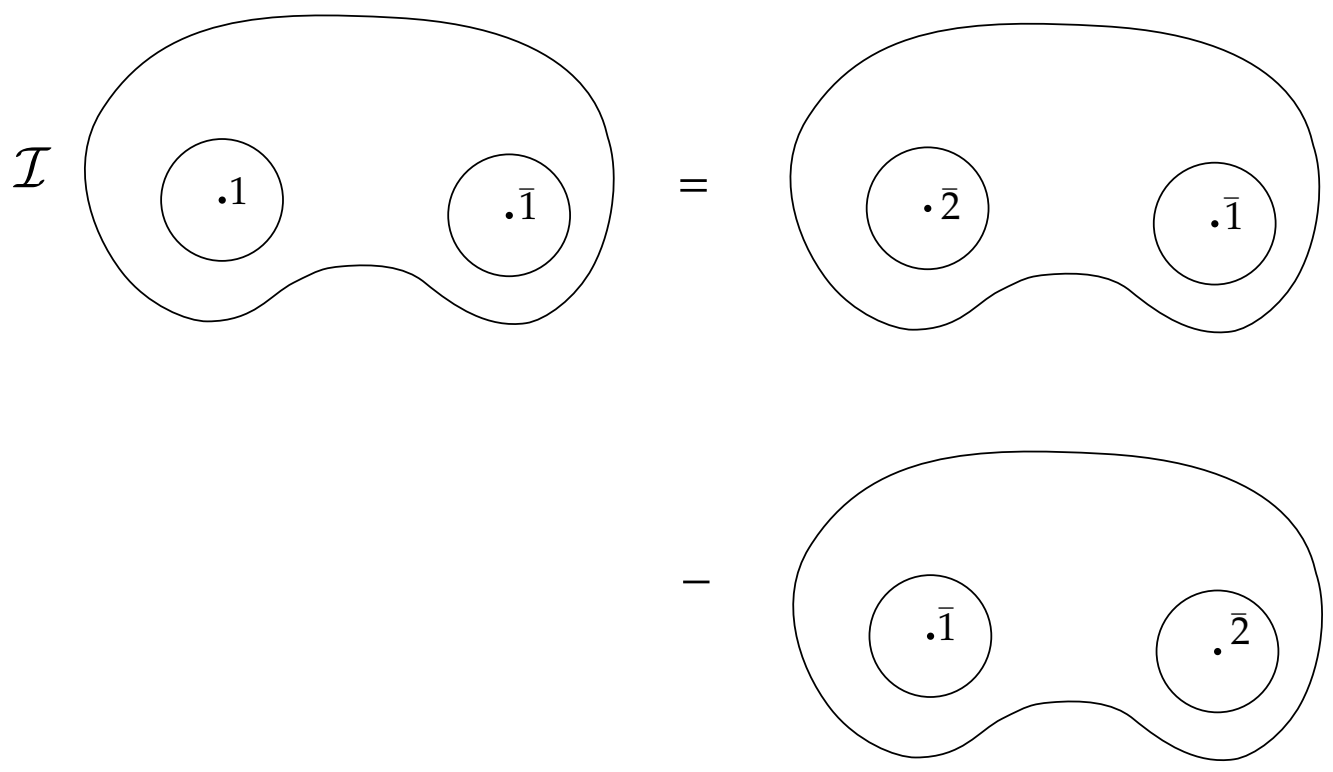

Figure 4. This figure illustrates the definition of the operator $\mathcal{I}$ when it acts on a surface with one ordinary puncture 1 and one special puncture $\overline{1}$. The ordinary puncture is made special and labelled $\overline{2}$. Then one antisymmetrizes on the special punctures.

The term $\mathcal{K} \mathcal{I}$ gives the surfaces where the original puncture is labeled $\overline{1}$ and the second puncture $\overline{2}$ moves throughout the surface, minus the surfaces with the labels exchanged. The term $\mathcal{I K}$ gives surfaces where the original puncture is labelled $\overline{2}$ and a puncture $\overline{1}$ moves on the surface minus the unit disk, minus the surfaces with the labels exchanged. It follows that the sum of terms gives surfaces where the original puncture is labelled $\overline{1}$ and the puncture $\overline{2}$ moves within the unit disk, minus the surfaces with the labels exchanged. These moduli spaces have an orientation which is that induced by the standard orientation of the disk where the puncture moves. Up to a sign this is precisely what the space $\mathcal{T}_{1}^{2}$ would do upon sewing via the antibracket.

Let us now justify the sign in the equation. It follows from the definition below (4.14) and the relation (4.15) that the surfaces with puncture $\overline{1}$ sitting and puncture $\overline{2}$ moving radially away appear in $\mathcal{T}_{1}^{2}$ with a minus sign. Call the radial vector $\partial / \partial r$. The twist sewing of the antibracket involves the rotation angle $\theta$ with the associated vector $\partial / \partial \theta$. The orientation of the right hand side is defined by the ordered basis $[\partial / \partial \theta, \partial / \partial r]$, which is opposite to the standard orientation of the disk. This induces a new minus sign, and we conclude that the overall sign must be a plus, as indicated in the equation. The extension to the case when we 


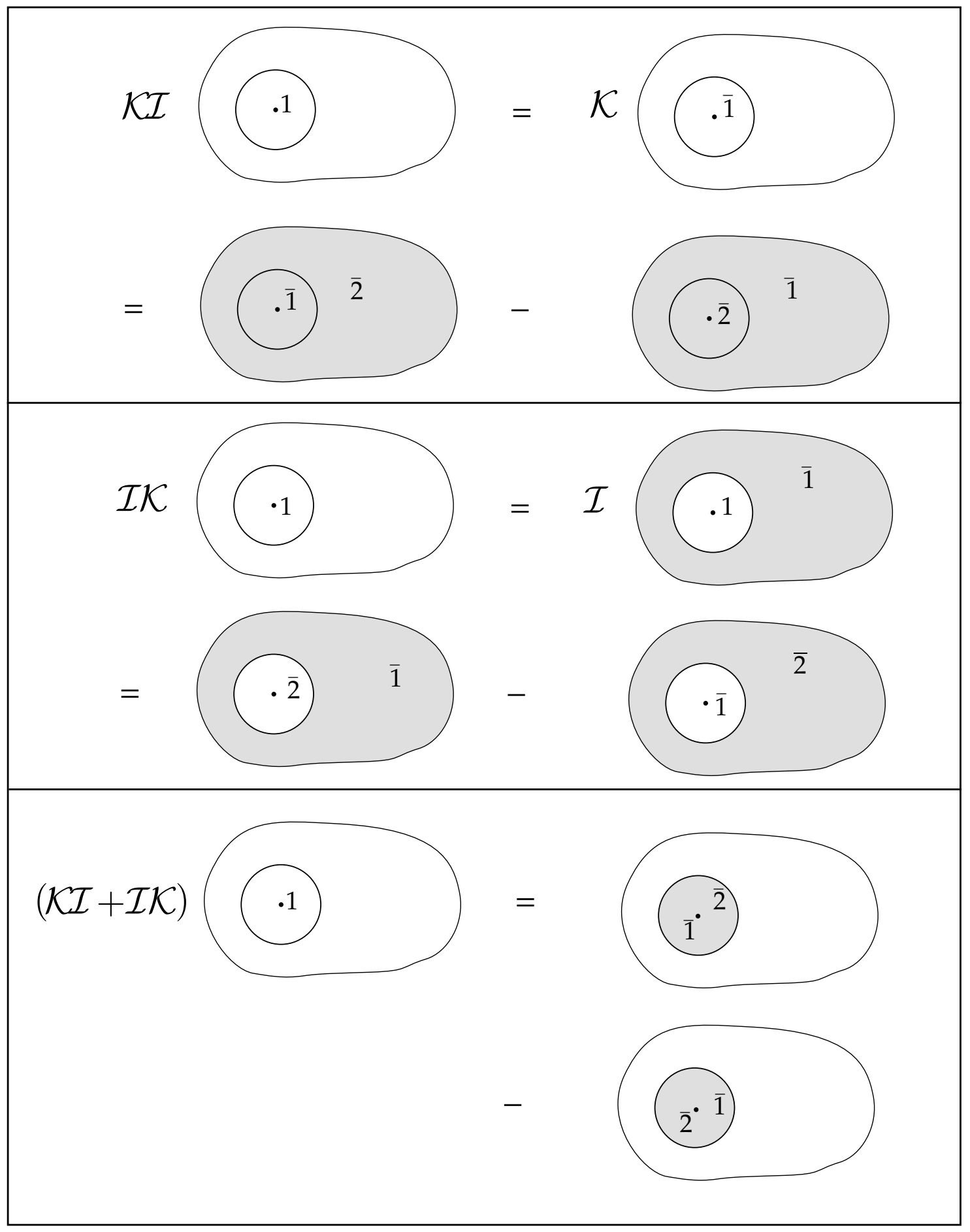

Figure 5. We illustrate the computation of $(\mathcal{K} \mathcal{I}+\mathcal{I K})$ acting on a surface with one ordinary puncture. The end result shows one of the special punctures sitting at the ordinary puncture, while the other one moves throughout the unit disk (with the usual antisymmetrization). 
act on $\mathcal{B}$ spaces is now immediate. Since the space $\mathcal{T}_{1}^{2}$ appears as the second entry in the antibracket, no new signs will be induced, the new special punctures will have the same labels in the left hand side and in the right hand side.

\subsection{The $\mathcal{M}$ operator.}

We now define $\mathcal{M} \equiv \mathcal{K}-\mathcal{I}$. This operator measures the difference between adding a special puncture and making an ordinary puncture special. We have given it a special name since it appears often enough. It follows from the identities given in (5.13) and (5.15) that

$$
\begin{aligned}
\mathcal{M}\left\{\mathcal{B}^{\bar{n}_{1}}, \mathcal{B}^{\bar{n}_{2}}\right\} & =(-)^{\mathcal{B}_{2}+\bar{n}_{2}+1}\left\{\mathcal{M B}^{\bar{n}_{1}}, \mathcal{B}^{\bar{n}_{2}}\right\}+\left\{\mathcal{B}^{\bar{n}_{1}}, \mathcal{M B}^{\bar{n}_{2}}\right\} \\
{[\partial, \mathcal{M}] \mathcal{B} } & =(-)^{\mathcal{B}+\bar{n}_{1}}\left\{\mathcal{V}_{3}^{\prime}, \mathcal{B}\right\} \\
\mathcal{M} \mathcal{M} & =-\left\{, \mathcal{T}_{1}^{2}\right\} .
\end{aligned}
$$

In deriving the last equation one makes use of (5.16).

It will be useful to bring in the operator $\delta_{\mathcal{V}}$ defined in (2.18). It is a short calculation to prove that

$$
\begin{aligned}
{\left[\delta_{\mathcal{V}}, \mathcal{K}\right] \mathcal{B} } & =(-)^{\mathcal{B}+\bar{n}}\left\{\mathcal{V}_{3}^{\prime}+\mathcal{K} \mathcal{V}, \mathcal{B}\right\} \\
{\left[\delta_{\mathcal{V}}, \mathcal{I}\right] \mathcal{B} } & =(-)^{\mathcal{B}+\bar{n}}\{\mathcal{I} \mathcal{V}, \mathcal{B}\} \\
{\left[\delta_{\mathcal{V}}, \mathcal{M}\right] \mathcal{B} } & =(-)^{\mathcal{B}+\bar{n}}\left\{\mathcal{V}_{3}^{\prime}+\mathcal{M} \mathcal{V}, \mathcal{B}\right\}
\end{aligned}
$$

\subsection{From Riemann surfaces to FunCtions}

We recall from Ref.[3] that there is a homomorphism from the BV algebra of Riemann surfaces to the BV algebra of functions in $\widehat{\mathcal{H}}$. One defines, for ordinary moduli spaces of surfaces

$$
f(\mathcal{A}) \equiv \frac{1}{n !} \int_{\mathcal{A}}\langle\Omega \mid \Psi\rangle_{1} \cdots|\Psi\rangle_{n}, \quad n \geq 1,
$$

and shows, using (2.10) and Eqn.(3.12) of Ref.[3] that

$$
f\left(\left\{\mathcal{A}_{1}, \mathcal{A}_{2}\right\}\right)=-\left\{f\left(\mathcal{A}_{1}\right), f\left(\mathcal{A}_{2}\right)\right\}
$$

For the action of the BRST operator one finds

$$
\{Q, f(\mathcal{A})\}=-f(\partial \mathcal{A}), \quad Q=\frac{1}{2}\left\langle\omega_{12}\left|\widehat{Q}^{(2)}\right| \Psi\right\rangle_{1}|\Psi\rangle_{2}
$$

We need to extend these results to the case when the moduli space of surfaces has special punctures. For moduli spaces with $n$ ordinary punctures and one or two special punctures 
respectively, we define

$$
\begin{aligned}
f_{\mathcal{O}_{1}}(\mathcal{B}) & \equiv \frac{1}{n !} \int_{\mathcal{B}}\langle\Omega \mid \Psi\rangle_{1} \cdots|\Psi\rangle_{n}\left|\mathcal{O}_{1}\right\rangle_{\overline{1}} \\
f_{\mathcal{O}_{1} \mathcal{O}_{2}}(\mathcal{B}) & \equiv \frac{1}{n !} \int_{\mathcal{B}}\langle\Omega \mid \Psi\rangle_{1} \cdots|\Psi\rangle_{n}\left|\mathcal{O}_{1}\right\rangle_{\overline{1}}\left|\mathcal{O}_{2}\right\rangle_{\overline{2}}
\end{aligned}
$$

Antibrackets between ordinary moduli spaces and special moduli spaces are readily found to map as

$$
\begin{aligned}
f_{\mathcal{O}_{1}}(\{\mathcal{A}, \mathcal{B}\}) & =-\left\{f(\mathcal{A}), f_{\mathcal{O}_{1}}(\mathcal{B})\right\} \\
f_{\mathcal{O}_{1} \mathcal{O}_{2}}(\{\mathcal{A}, \mathcal{B}\}) & =-\left\{f(\mathcal{A}), f_{\mathcal{O}_{1} \mathcal{O}_{2}}(\mathcal{B})\right\}
\end{aligned}
$$

Note that given (5.7) there is no difference between $\{\mathcal{A}, \mathcal{B}\}^{\prime}$ and $\{\mathcal{A}, \mathcal{B}\}$. We can thus justify (5.23) using the primed antibracket, and then simply use the unprimed one.

The antibracket between two moduli spaces each of one special puncture is given by

$$
f_{i j}\left(\left\{\mathcal{B}, \mathcal{B}^{\prime}\right\}^{\prime}\right)=(-)^{1+i\left(\mathcal{B}^{\prime}+1\right)}\left[\left\{f_{i}(\mathcal{B}), f_{j}\left(\mathcal{B}^{\prime}\right)\right\}+(-)^{(i+1)(j+1)+\mathcal{B}^{\prime}(i+j)}\left\{f_{j}(\mathcal{B}), f_{i}\left(\mathcal{B}^{\prime}\right)\right\}\right] .
$$

Here we use subscripts $i$ and $j$ instead of $\mathcal{O}_{i}$ and $\mathcal{O}_{j}$, for the sake of brevity. The right hand side shows two terms, which arise because the antibracket in the left hand side involves an antisymmetrization on the special punctures (see sect.4). The sign factors have been given in all generality. If the states are both grassmann even, the above equation gives

$$
f_{\mu \nu}\left(\left\{\mathcal{B}, \mathcal{B}^{\prime}\right\}^{\prime}\right)=-\left\{f_{\mu}(\mathcal{B}), f_{\nu}\left(\mathcal{B}^{\prime}\right)\right\}+\left\{f_{\nu}(\mathcal{B}), f_{\mu}\left(\mathcal{B}^{\prime}\right)\right\}
$$

If, in addition, $\mathcal{B}=\mathcal{B}^{\prime}$, and $\operatorname{dim}(\mathcal{B})$ is odd we find (see (5.7))

$$
f_{\mu \nu}(\{\mathcal{B}, \mathcal{B}\})=-2\left\{f_{\mu}(\mathcal{B}), f_{\nu}(\mathcal{B})\right\}
$$

where we now use the unprimed antibracket in the left hand side. This equation holds, by virtue of (5.25), when the space $\mathcal{B}$ is actually a sum of spaces, all with one special puncture and all odd-dimensional.

Let us now consider the homomorphism identities relevant to the operator $\mathcal{I}$. The linear hamiltonian $B_{\mathcal{O}}^{(2)}$ (formerly called $U_{\mathcal{O}}^{(2)}$ ) is the hamiltonian required to produce insertions of 
special states on ordinary punctures

$$
B_{\mathcal{O}}^{(2)} \equiv\left\langle\omega_{12} \mid \mathcal{O}\right\rangle_{1}|\Psi\rangle_{2}
$$

One readily finds that the antibracket of two such hamiltonians is a constant hamiltonian

$$
\left\{B_{\mathcal{O}_{1}}^{(2)}, B_{\mathcal{O}_{2}}^{(2)}\right\}=\left\langle\omega_{12} \mid \mathcal{O}_{1}\right\rangle_{1}\left|\mathcal{O}_{2}\right\rangle_{2}
$$

Inserting states on moduli spaces of surfaces with ordinary punctures is straightforward

$$
f_{\mathcal{O}}(\mathcal{I} \mathcal{A})=\left\{f(\mathcal{A}), B_{\mathcal{O}}^{(2)}\right\} .
$$

To insert a state on a moduli space of surfaces that already has one special puncture, we recall that the operator $\mathcal{I}$ will convert an ordinary puncture into a special one and then will antisymmetrize on the special punctures. One finds

$$
f_{i j}(\mathcal{I} \mathcal{B})=\left\{f_{i}(\mathcal{B}), B_{j}^{(2)}\right\}+(-)^{i(\mathcal{B}+1)}\left\{B_{i}^{(2)}, f_{j}(\mathcal{B})\right\},
$$

where again, for brevity, we $i$ and $j$ instead of $\mathcal{O}_{i}$ and $\mathcal{O}_{j}$. For grassmann even states the above simply becomes

$$
f_{\mu \nu}(\mathcal{I B})=\left\{f_{\mu}(\mathcal{B}), B_{\nu}^{(2)}\right\}+\left\{B_{\mu}^{(2)}, f_{\nu}(\mathcal{B})\right\} .
$$

BRST action on ordinary moduli spaces is familiar (see, for example [3])

$$
\{Q, f(\mathcal{A})\}=-f(\partial \mathcal{A}) .
$$

If we act on a $\mathcal{B}$ space with one special puncture we find

$$
\left\{Q, f_{\mathcal{O}}(\mathcal{B})\right\}=-f_{\mathcal{O}}(\partial \mathcal{B})+(-)^{\mathcal{B}} f_{Q \mathcal{O}}(\mathcal{B}),
$$

by doing a computation virtually identical to that used to establish Eqn.(2.41) of Ref.[21]. For $\mathcal{B}$ spaces with two or more punctures there is only little difference

$$
\left\{Q, f_{\mathcal{O}_{i} \mathcal{O}_{j}}(\mathcal{B})\right\}=-f_{\mathcal{O}_{i} \mathcal{O}_{j}}(\partial \mathcal{B})+(-)^{\mathcal{B}}\left[f_{\left(Q \mathcal{O}_{i}\right) \mathcal{O}_{j}}+(-)^{i} f_{\mathcal{O}_{i} Q \mathcal{O}_{j}}\right](\mathcal{B}),
$$

For our case of interest we will look at $f_{\mu \nu}=f_{\widehat{\mathcal{O}}_{\mu} \widehat{\mathcal{O}}_{\nu}}$ and since both $\widehat{\mathcal{O}}_{\mu}$ and $\widehat{\mathcal{O}}_{\nu}$ are annihilated by $Q$, we find

$$
\begin{aligned}
\left\{S, f_{\mu \nu}(\mathcal{B})\right\} & =\left\{Q+f(\mathcal{V}), f_{\mu \nu}(\mathcal{B})\right\} \\
& =-f_{\mu \nu}(\partial \mathcal{B}+\{\mathcal{V}, \mathcal{B}\}) \\
& =-f_{\mu \nu}\left(\delta_{\mathcal{V}} \mathcal{B}\right)
\end{aligned}
$$

where use was made of (5.23). 
With the canonical connection, covariant derivatives of hamiltonians arising from ordinary moduli spaces are readily computed [3]

$$
D_{\mu} f(\mathcal{A})=f_{\mu}(\mathcal{K} \mathcal{A})
$$

For moduli spaces with one special puncture filled with a marginal operator $\left(\widehat{\mathcal{O}}_{\mu}, \widehat{\mathcal{O}}_{\nu}, \cdots\right)$, antisymmetric covariant derivatives take a simple form. Consider the covariant derivative $D_{\mu} f_{\nu}(\mathcal{B})$. It follows from the first equation in (5.22) that there are two contributions, one from the derivative of the surface state and the other from the derivative of $\left|\widehat{\mathcal{O}}_{\nu}\right\rangle$. We find

$$
D_{\mu} f_{\nu}(\mathcal{B})=f_{\nu \mu}(\widetilde{\mathcal{K}} \mathcal{B})+f_{D_{\mu}\left|\widehat{\mathcal{O}}_{\nu}\right\rangle}(\mathcal{B})
$$

where $\widetilde{\mathcal{K}}$ denotes operation of adding a puncture, labelled $\overline{2}$, where $\widehat{\mathcal{O}}_{\mu}$ is inserted, avoiding all coordinate disks including that of the special puncture. Note that $\widetilde{\mathcal{K}}$ performs no antisymmetrization. Forming the antisymmetric combination we find

$$
D_{\mu} f_{\nu}(\mathcal{B})-D_{\nu} f_{\mu}(\mathcal{B})=f_{\nu \mu-\mu \nu}(\widetilde{\mathcal{K}} \mathcal{B})+f_{D_{\mu}\left|\widehat{\mathcal{O}}_{\nu}\right\rangle-D_{\nu}\left|\widehat{\mathcal{O}}_{\mu}\right\rangle}(\mathcal{B})
$$

Using (4.2), we recognize that the second term in the right hand side is making an insertion throughout the disk associated with the special puncture. In fact, the signs work out correctly (compare (4.2) with (2.14)) so that the two terms in the right hand side combine to make insertions throughout the surface avoiding only the disks associated with ordinary punctures. This is precisely the operation $\mathcal{K}$, which includes antisymmetrization. We have therefore shown that

$$
D_{\mu} f_{\nu}(\mathcal{B})-D_{\nu} f_{\mu}(\mathcal{B})=-f_{\mu \nu}(\mathcal{K} \mathcal{B})
$$




\section{Consistency Conditions for Moduli Spaces}

In this section we will examine explicitly the consistency conditions derived in section 3 . They read

$$
D_{\mu} B_{\nu}-D_{\nu} B_{\mu}+\left\{B_{\mu}, B_{\nu}\right\}+R_{\mu \nu}=\left\{S, B_{\mu \nu}\right\}
$$

Since the above covariant derivatives are taken with respect to the canonical connection $\Gamma_{\mu}$ and this connection has vanishing curvature $R_{\mu \nu}(\Gamma)=0$, the equation to be discussed is

$$
D_{\mu} B_{\nu}-D_{\nu} B_{\mu}+\left\{B_{\mu}, B_{\nu}\right\}=\left\{S, B_{\mu \nu}\right\}
$$

The aim of the present section is to show that the hamiltonian $B_{\mu \nu}$ is the function associated to some moduli spaces of surfaces with two special punctures. We will use our analysis of the previous section to derive the recursion relations that must be satisfied by the moduli spaces that define the hamiltonian $B_{\mu \nu}$.

We therefore take $B_{\mu \nu}$ to be a hamiltonian of the form

$$
B_{\mu \nu}=-f_{\mu \nu}\left(\mathcal{B}^{2}\right)=-\int_{\mathcal{B}^{2}}\left\langle\Omega_{\overline{1} \overline{2}} \mid \mathcal{O}_{\mu}\right\rangle_{\overline{1}}\left|\mathcal{O}_{\mu}\right\rangle_{\overline{2}},
$$

where $\mathcal{B}^{2}$ is a sum of moduli spaces of surfaces with two special punctures. The spaces in $\mathcal{B}^{2}$ are antisymmetric under the exchange of labels of the special punctures, $\left\langle\Omega_{\overline{1} \overline{2}}\right|=-\left\langle\Omega_{\overline{2} \overline{1}}\right|$, and this explains why $B_{\mu \nu}=-B_{\nu \mu}$.

The right hand side of (6.2) is readily written in terms of moduli spaces

$$
\left\{S, B_{\mu \nu}\right\}=-\left\{S, f_{\mu \nu}\left(\mathcal{B}^{2}\right)\right\}=f_{\mu \nu}\left(\delta_{\mathcal{V}} \mathcal{B}^{2}\right)
$$

where use was made of Eqn.(5.35). Let us now compute $\left(D_{\mu} B_{\nu}-D_{\nu} B_{\mu}\right)$. Since $B_{\nu}=$ $B_{\nu}^{(2)}-f_{\nu}\left(\mathcal{B}^{1}\right)$, we must consider

$$
D_{\mu} B_{\nu}-D_{\nu} B_{\mu}=\left(D_{\mu} B_{\nu}^{(2)}-D_{\nu} B_{\mu}^{(2)}\right)-\left(D_{\mu} f_{\nu}\left(\mathcal{B}^{1}\right)-D_{\nu} f_{\mu}\left(\mathcal{B}^{1}\right)\right)
$$

The first parenthesis is readily computed

$$
D_{\mu} B_{\nu}^{(2)}-D_{\nu} B_{\mu}^{(2)}=B_{D_{\mu}\left|\mathcal{O}_{\nu}\right\rangle-D_{\nu}\left|\mathcal{O}_{\mu}\right\rangle}^{(2)}=f_{\mu \nu}\left(\mathcal{T}_{1}^{2}\right)
$$

where we made use of (4.31). The second parenthesis was computed in (5.39), so all together 
we find

$$
D_{\mu} B_{\nu}-D_{\nu} B_{\mu}=f_{\mu \nu}\left(\mathcal{T}_{1}^{2}+\mathcal{K} \mathcal{B}^{1}\right)
$$

On the other hand

$$
\begin{aligned}
\left\{B_{\mu}, B_{\nu}\right\}= & \left\{B_{\mu}^{(2)}, B_{\nu}^{(2)}\right\} \\
& -\left\{f_{\mu}\left(\mathcal{B}^{1}\right), B_{\nu}^{(2)}\right\}-\left\{B_{\mu}^{(2)}, f_{\nu}\left(\mathcal{B}^{1}\right)\right\} \\
& +\left\{f_{\mu}\left(\mathcal{B}^{1}\right), f_{\nu}\left(\mathcal{B}^{1}\right)\right\}
\end{aligned}
$$

The first line in the right hand side vanishes by virtue of (5.28), the second line is simplified using (5.30) and the third line using (5.26). We thus find

$$
\left\{B_{\mu}, B_{\nu}\right\}=-f_{\mu \nu}\left(\mathcal{I B}^{1}+\frac{1}{2}\left\{\mathcal{B}^{1}, \mathcal{B}^{1}\right\}\right) .
$$

It is now possible to put together Eqns.(6.4),(6.7), and (6.9) to find

$$
f_{\mu \nu}\left(\mathcal{T}_{1}^{2}+\mathcal{M} \mathcal{B}^{1}-\frac{1}{2}\left\{\mathcal{B}^{1}, \mathcal{B}^{1}\right\}\right)=f_{\mu \nu}\left(\delta_{\mathcal{\nu}} \mathcal{B}^{2}\right) .
$$

This equation will be satisfied if

$$
\delta_{\mathcal{V}} \mathcal{B}^{2}=\mathcal{T}_{1}^{2}+\mathcal{M} \mathcal{B}^{1}-\frac{1}{2}\left\{\mathcal{B}^{1}, \mathcal{B}^{1}\right\} .
$$

Note that by construction the surfaces in the right hand side of the equation are all $\mathcal{B}^{2}$ spaces, namely moduli spaces of surfaces with two special punctures, antisymmetric under the exchange of those punctures. The antisymmetry of the hamiltonian $B_{\mu \nu}$ implied that $\mathcal{B}^{2}$ had to be a $\mathcal{B}$ space, and the action of $\delta_{\mathcal{V}}$ does not change the fact that it is a $\mathcal{B}$ space. Thus the above equation is an equation relating $\mathcal{B}$ spaces.

Let us now verify the consistency of (6.11). We should be able to check that $\delta_{\mathcal{V}} \delta_{\mathcal{V}} \mathcal{B}^{2}=0$. We calculate the action of $\delta \mathcal{V}$ on each term of the right hand side of (6.11) and find

$$
\begin{aligned}
\delta_{\mathcal{V}} \mathcal{T}_{1}^{2} & =\partial \mathcal{T}_{1}^{2}+\left\{\mathcal{V}, \mathcal{T}_{1}^{2}\right\}=\mathcal{I} \mathcal{V}_{3}^{\prime}+\left\{\mathcal{V}, \mathcal{T}_{1}^{2}\right\} \\
\delta \mathcal{V} \mathcal{M} \mathcal{B}^{1} & =[\delta \mathcal{V}, \mathcal{M}] \mathcal{B}^{1}+\mathcal{M} \delta_{\mathcal{V}} \mathcal{B}^{1} \\
& =\left\{\mathcal{V}_{3}^{\prime}+\mathcal{M V}, \mathcal{B}^{1}\right\}+\mathcal{M}\left(\mathcal{V}_{3}^{\prime}+\mathcal{M V}\right) \\
& =\left\{\mathcal{V}_{3}^{\prime}+\mathcal{M V}, \mathcal{B}^{1}\right\}-\mathcal{I} \mathcal{V}_{3}^{\prime}-\left\{\mathcal{V}, \mathcal{T}_{1}^{2}\right\} \\
\delta_{\mathcal{V}}\left(-\frac{1}{2}\left\{\mathcal{B}^{1}, \mathcal{B}^{1}\right\}\right) & =-\left\{\delta_{\mathcal{V}} \mathcal{B}^{1}, \mathcal{B}^{1}\right\}=-\left\{\mathcal{V}_{3}^{\prime}+\mathcal{M V}, \mathcal{B}^{1}\right\}
\end{aligned}
$$

Back in (6.11) we see that all terms cancel out, and thus the recursion relations are consistent. 
To derive the first equation we used (4.18), and for the second we used (5.18), (2.23), (5.8), and (5.17). We must now show that we can actually define the $\mathcal{B}^{2}$ spaces of the left hand side of (6.11). This is the subject of the next section.

\section{Recursive construction of $\mathcal{B}^{2}$ spaces}

In order to construct the $\mathcal{B}^{2}$ spaces we must write the consistency condition (6.11) as a recursion relation. To this end we rewrite this equation as

$$
\partial \mathcal{B}^{2}=\mathcal{T}_{1}^{2}+\mathcal{M} \mathcal{B}^{1}-\frac{1}{2}\left\{\mathcal{B}^{1}, \mathcal{B}^{1}\right\}-\left\{\mathcal{V}, \mathcal{B}^{2}\right\}
$$

It is worthwhile noticing that the consistency check $\delta_{\mathcal{V}}^{2} \mathcal{B}^{2}=0$ we performed in the previous section is equivalent to checking that $\partial \partial \mathcal{B}^{2}=0$. This establishes that the right hand side of (7.1) indeed has zero boundary, a necessary, though not sufficient condition for it to be a boundary. Showing it is a boundary is the purpose of the present section. Our analysis will be very explicit for the $\mathcal{B}^{2}$ spaces with the lowest number of ordinary punctures. We then outline a construction for the $\mathcal{B}^{2}$ spaces with higher number of ordinary punctures.

It is straightforward to see that (7.1) is a set of recursion relations. Equation (7.1) gives an equation for each number of ordinary punctures. All $\mathcal{B}^{2}$ spaces have two special punctures, and we will define them recursively on the number of ordinary punctures. For any chosen number $k$ of ordinary punctures $\partial \mathcal{B}_{k}^{2}$ involves either known spaces, $\left(\mathcal{B}^{1}\right.$ or $\mathcal{V}$ spaces) or $\mathcal{B}_{k^{\prime}}^{2}$ spaces with $k^{\prime}<k$, given that $\mathcal{V}$ has at least three ordinary punctures and thus the antibracket adds at least two ordinary punctures. The lowest number of ordinary punctures appearing in the right hand side is one. Therefore we will have

$$
\mathcal{B}^{2} \equiv \mathcal{B}_{1}^{2}+\mathcal{B}_{2}^{2}+\mathcal{B}_{3}^{2}+\cdots
$$

The first two equations that follow from (7.1) are

$$
\begin{aligned}
& \partial \mathcal{B}_{1}^{2}=\mathcal{T}_{1}^{2}-\mathcal{I} \mathcal{B}_{2}^{1} \\
& \partial \mathcal{B}_{2}^{2}=\mathcal{K} \mathcal{B}_{2}^{1}-\mathcal{I B}_{3}^{1}-\frac{1}{2}\left\{\mathcal{B}_{2}^{1}, \mathcal{B}_{2}^{1}\right\}-\left\{\mathcal{V}_{3}, \mathcal{B}_{1}^{2}\right\}
\end{aligned}
$$

Let us first discuss explicitly the first equation. 


\subsection{Construction of $\mathcal{B}_{1}^{2}$}

We want to construct the space $\mathcal{B}_{1}^{2}$ that satisfies $\partial \mathcal{B}_{1}^{2}=\mathcal{T}_{1}^{2}-\mathcal{I B}_{2}^{1}$. We recall from (4.18) and (2.22) that

$$
\partial \mathcal{T}_{1}^{2}=\mathcal{I} \mathcal{V}_{3}^{\prime}, \quad \partial\left(\mathcal{I} \mathcal{B}_{2}^{1}\right)=\mathcal{I} \partial \mathcal{B}_{2}^{1}=\mathcal{I}\left(\mathcal{V}_{3}^{\prime}-\mathcal{I V}_{3}\right)=\mathcal{I} \mathcal{V}_{3}^{\prime}
$$

These two relations explain that $\partial^{2} \mathcal{B}_{1}^{2}=0$, but in addition they help us understand the geometrical picture, as is shown in Fig.6. It is useful to consider the primitive domains of the moduli spaces $\mathcal{T}_{1}^{2}$ and $\mathcal{I B}_{2}^{1}$ :

- The primitive domain $\overline{\mathcal{T}_{1}^{2}}$ is the set of surfaces $I S(t)$ ( $I$ is the same as $\mathcal{I}$ except that it does not include antisymmetrization on the special punctures) with $t \in[0,1]$, and thus extending from the sphere $I S(0)$ up to $I \mathcal{V}_{3}^{\prime}$. Let $\overline{\mathcal{T}_{1}^{2}}\left[t_{0}\right]$ denote the particular three-punctured sphere obtained for $t=t_{0}$.

- The primitive domain $\overline{\mathcal{I} \mathcal{B}_{2}^{1}}$ is the set of surfaces interpolating from $I I \mathcal{V}_{3}$ up to $I \mathcal{V}_{3}^{\prime}$, a set that can also be parametrized by $t \in[0,1]$. Similarly, let $\overline{\mathcal{I B}_{2}^{1}}\left[t_{0}\right]$ denote the particular three-punctured sphere obtained for $t=t_{0}$.

The two primitive spaces meet at the surface $I \mathcal{V}_{3}^{\prime}$. Moreover

$$
\partial\left(\overline{\mathcal{T}_{1}^{2}}-\overline{\mathcal{I B}_{2}^{1}}\right)=I S(0)-I I \mathcal{V}_{3}
$$

and thus the primitive domain of the space in the right hand side of the recursion relation extends from the totally symmetric three punctured sphere $I I \mathcal{V}_{3}$ to the sphere $I S(0)$, which is symmetric in the two special punctures it has (the two punctures actually coincide). Recall that all surfaces involved here are three punctured spheres and therefore they only differ by the choice of local coordinates at their punctures.

- Let $\Sigma\left(s, t_{0}\right)$ with $s \in[0,1]$ denote the set of spheres interpolating from $\Sigma\left(0, t_{0}\right)=\overline{\mathcal{I} \mathcal{B}_{2}^{1}}\left[t_{0}\right]$ up to $\Sigma\left(1, t_{0}\right)=\overline{\mathcal{T}_{1}^{2}}\left[t_{0}\right]$. All interpolations are done canonically (as in Ref.[ 2] Sect.3.4).

We now define the object $\overline{\mathcal{B}_{1}^{2}}$

$$
\overline{\mathcal{B}_{1}^{2}} \equiv\{\Sigma(s, t) \mid s, t \in[0,1]\}
$$

with orientation defined by the ordered basis $\left[\frac{\partial}{\partial s}, \frac{\partial}{\partial t}\right]$. It follows from this definition that

$$
\partial \overline{\mathcal{B}_{1}^{2}}=\overline{\mathcal{T}_{1}^{2}}-\overline{\mathcal{I} \mathcal{B}_{2}^{1}}+\Sigma(s, 0),
$$

where the last term denotes the surfaces interpolating canonically from the symmetric vertex 


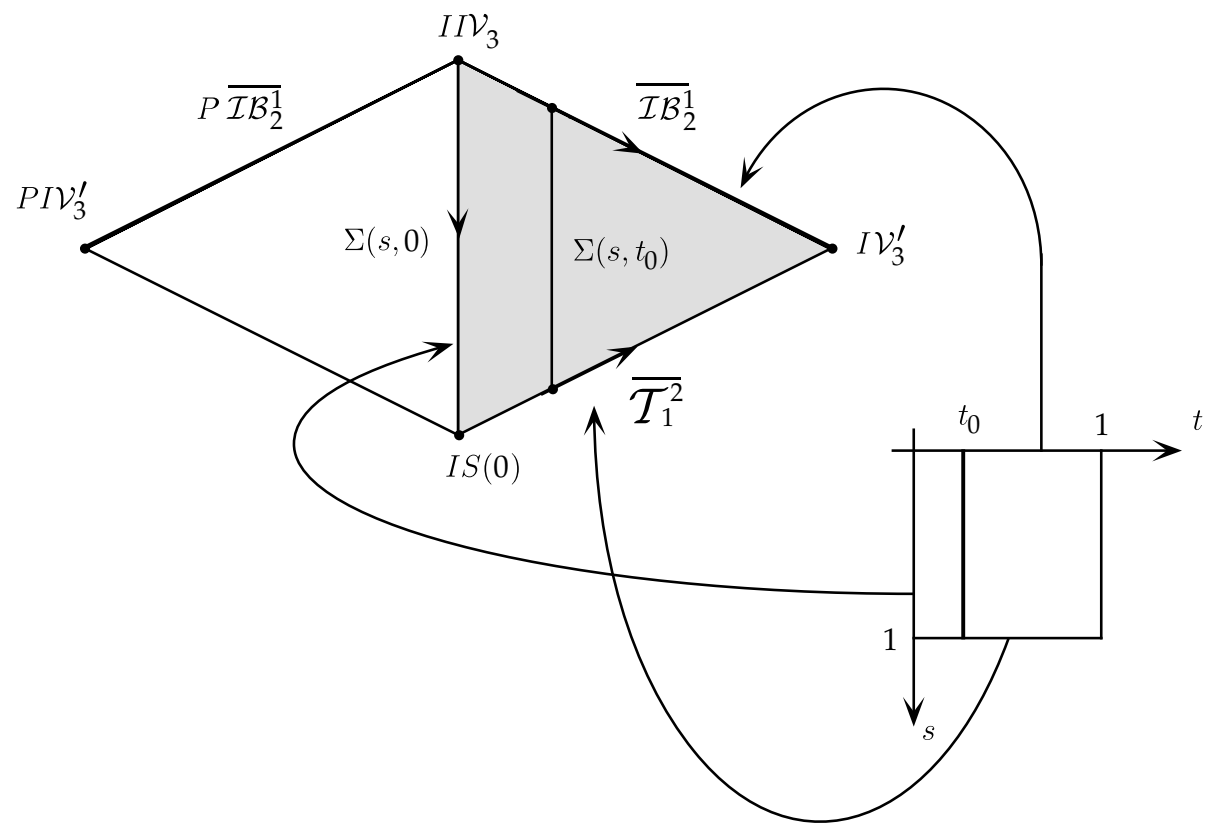

Figure 6. The rhombus shaped region represents the desired space $\mathcal{B}_{1}^{2}$. Its primitive domain is shown shaded. The boundary of the primitive domain is $\overline{\mathcal{T}_{1}^{2}}-\overline{\mathcal{I} \mathcal{B}_{2}^{1}}$ plus the set of surfaces $\Sigma(s, 0)$ interpolating symmetrically from $I I \mathcal{V}_{3}$ up to $I S(0)$.

$I I \mathcal{V}_{3}$ up to the symmetric vertex $I S(0)$. It follows from the nature of canonical interpolation that all surfaces in $\Sigma(s, 0)$ are symmetric under the exchange of the two special punctures. Note that there is no contribution to the boundary from $\Sigma(s, 1)$ since it is an interpolation between identical surfaces $\left(I \mathcal{V}_{3}^{\prime}\right)$. We claim that, as the notation suggests, $\overline{\mathcal{B}_{1}^{2}}$ is the fundamental domain of the desired space $\mathcal{B}_{1}^{2}$, namely

$$
\mathcal{B}_{1}^{2}=\overline{\mathcal{B}_{1}^{2}}-P \overline{\mathcal{B}_{1}^{2}}
$$

We verify this by taking now the boundary, and using $P \partial=\partial P$, together with (7.7), to find

$$
\begin{aligned}
\partial \mathcal{B}_{1}^{2} & =(1-P) \partial \overline{\mathcal{B}_{1}^{2}} \\
& =\mathcal{T}_{1}^{2}-\mathcal{I B}_{2}^{1}+(\Sigma(s, 0)-P \Sigma(s, 0)) \\
& =\mathcal{T}_{1}^{2}-\mathcal{I B}_{2}^{1}
\end{aligned}
$$

using the symmetry of the space $\Sigma(s, 0)$. Having shown that the $\mathcal{B}_{1}^{2}$ space defined by $(7.7)$ and (7.8) has the correct boundary this completes our analysis of the first consistency condition. 


\subsection{Iterative CONSTRUCtion OF THE $\mathcal{B}_{k}^{2}$ SPACES}

The second recursion relation listed in (7.3) is an example of the general situation which takes the form

$$
\partial \mathcal{B}_{k}^{2}=\mathcal{K} \mathcal{B}_{k}^{1}-\left(\mathcal{I B}_{k+1}^{1}+\cdots\right)
$$

By the consistency condition, the space $\mathcal{K} \mathcal{B}_{k}^{1}$, to be called $\mathcal{C}$, and the second space, indicated by parenthesis and to be called $\mathcal{C}^{\prime}$, are two $\mathcal{B}^{2}$ spaces having identical boundaries (the specific way of splitting the right hand side of the recursion relation will not be relevant). Our problem is to construct an interpolation between the $\mathcal{B}^{2}$ spaces $\mathcal{C}$ and $\mathcal{C}^{\prime}$, such that the boundary of the interpolation gives the two spaces in question. Note that the surfaces involved are all spheres with $k+2$ punctures, and the spaces $\mathcal{C}$ and $\mathcal{C}^{\prime}$ have real dimension one higher than that of the moduli space $\mathcal{M}_{k+2}$ of punctured Riemann spheres without decoration. Thus each of the two $\mathcal{B}^{2}$ spaces can be thought as built from one dimensional fibers over $\mathcal{M}_{k+2}$.

The idea now is to project $\mathcal{C}$, which sits in $\widehat{\mathcal{P}}_{k+2}$ (the moduli space of $(k+2)$-punctured spheres having local coordinates at the punctures) down to the base $\mathcal{M}_{k+2}$ via the projection $\pi: \widehat{\mathcal{P}}_{k+2} \rightarrow \mathcal{M}_{k+2}$. We then to examine for each point $p \in \pi(\mathcal{C})$ the intersection $\pi^{-1}(p) \cap \mathcal{C} \equiv$ $\mathcal{C}_{p}$, namely the intersection of the (infinite dimensional) fiber over $p$ with the space $\mathcal{C}$. This intersection $\mathcal{C}_{p}$ must be a one dimensional subspace of $\mathcal{C}$, since the dimensionality of $\mathcal{C}$ exceeds that of $\mathcal{M}_{k+2}$ by one. We choose some consistent parametrization of the surfaces on each of the spaces $\mathcal{C}_{p}$; namely, they are parametrized by a $t \in[0,1]$ such that for nearby points $p, p^{\prime}$ and nearby parameters $t, t^{\prime}$ we get nearby surfaces, and the parametrization is compatible with the antisymmetry properties on the exchange of special punctures. The space $\mathcal{C}$ can be reconstructed as the union over all $p$ of the spaces $\mathcal{C}_{p}$, namely $\mathcal{C}=\cup_{p} \mathcal{C}_{p}$.

The one dimensional space $\mathcal{C}_{p}$ must have two endpoints on $\partial \mathcal{C}^{\star}$. The space $\mathcal{C}_{p}^{\prime} \equiv \pi^{-1}(p) \cap \mathcal{C}^{\prime}$ must also have endpoints on $\partial \mathcal{C}_{p}^{\prime}$, and the endpoints of $\mathcal{C}_{p}$ and $\mathcal{C}_{p}^{\prime}$ must coincide given that $\partial\left(\mathcal{C}-\mathcal{C}^{\prime}\right)=0$, and $\partial\left(\mathcal{C}-\mathcal{C}^{\prime}\right)=\cup_{p}\left(\partial \mathcal{C}_{p}-\partial \mathcal{C}_{p}^{\prime}\right)$. We now construct a space $\mathcal{D}_{p}$ interpolating between the parametrized spaces $\mathcal{C}_{p}$ and $\mathcal{C}_{p}^{\prime}$ by canonical interpolation on the local coordinates at the punctures for each value of the parameter. This interpolation is all that needs to be done since all the surfaces agree as punctured Riemann surfaces without decoration. Note that by construction the boundary of $\mathcal{D}_{p}$ is simply $\mathcal{C}_{p}-\mathcal{C}_{p}^{\prime}$. The space $\mathcal{D}_{p}$ is built for all points $p$,

\footnotetext{
$\star$ It is possible, in principle, that $\mathcal{C}_{p}$ takes the form of a disjoint union of segments, each with endpoints on $\partial \mathcal{C}$. In this case one would use several parameters, all running from zero to one.
} 
and we take $\mathcal{B}_{k}^{2}=\cup_{p} \mathcal{D}_{p}$. It follows that $\partial \mathcal{B}_{k}^{2}=\cup_{p} \partial \mathcal{D}_{p}=\cup_{p}\left(\mathcal{C}_{p}-\mathcal{C}_{p}^{\prime}\right)=\mathcal{C}-\mathcal{C}^{\prime}$, as desired. This completes the construction.

Acknowledgements: I wish to thank R. Cohen for preparing the figures.

\section{REFERENCES}

1. A. Sen and B. Zwiebach, 'Background independent algebraic structures in closed string field theory', MIT-CTP-2346, August 1994, hep-th/9408053.

2. A. Sen and B. Zwiebach, 'Local background independence of classical closed string field theory', Nucl. Phys. B414 (1994) 649, hep-th/9307088.

3. A. Sen and B. Zwiebach, 'Quantum background independence of closed string field theory', Nucl. Phys. B423 (1994) 580, hep-th/9311009.

4. A. Sen and B. Zwiebach, 'A note on gauge transformations in Batalin-Vilkovisky theory', Phys. Lett. B320 (1994) 29, hep-th/9309027.

5. T. Kimura, J. Stasheff, and A. A. Voronov, 'On operad structures of moduli spaces and string theory', Commun. Math. Phys. 171 (1995) 1, hep-th/9307114;

'Homology of moduli spaces of curves and commutative homotopy algebras' , alggeom/9502006.

6. T. Kimura and A. A. Voronov, 'The cohomology of algebras over moduli spaces', University of North Carolina preprint, October 1994, hep-th/9410108.

7. B. Zwiebach, 'Closed string field theory: Quantum action and the Batalin-Vilkovisky master equation', Nucl. Phys B390 (1993) 33, hep-th/9206084.

8. E. Getzler, 'Batalin-Vilkovisky algebras and two-dimensional topological field theories', Commun. Math. Phys. 159 (1994) 265; hep-th/9212043.

9. B. H. Lian and G. Zuckerman, "New Perspectives on the BRST-algebraic structure of string theory", Commun. Math. Phys. 154 (1993) 613, hep-th/9211072.

10. M. Penkava and A. Schwarz, "On Some Algebraic Structures Arising in String Theory", UC Davis preprint, UCD-92-03, hep-th/9212072.

11. K. Bering, P.H. Damgaard, and J. Alfaro, 'Algebra of higher antibrackets' hepth/9604027.

12. F. Akman, 'On some generalizations of Batalin-Vilkovisky algebras', q-alg/9506027 
13. M. Campbell, P. Nelson and E. Wong, 'Stress tensor perturbations in conformal field theory', Int. Jour. Mod. Phys. A6 (1991) 4909.

14. K. Ranganathan, 'Nearby CFT's in the operator formalism: the role of a connection', Nucl. Phys. B408 (1993) 180.

15. K. Ranganathan, H. Sonoda and B. Zwiebach, 'Connections on the state-space over conformal field theories', Nucl. Phys. B414 (1994) 405, hep-th/9304053.

16. H. Sonoda, Composite operators in QCD, Nucl. Phys. B383 (1992) 173, hep-th/9205085; "Operator Coefficients for Composite Operators in the $\left(\phi^{4}\right)_{4}$ Theory", Nucl. Phys. B394 (1993) 302, hep-th/9205084.

17. A. Gussich, and P. Sundell, 'Finite deformations of conformal field theories using analytically regularized connections', Göteborg preprint, ITP 96-5, hep-th/9604010.

18. B. Zwiebach, 'On the construction of string field theory around non-conformal backgrounds', MIT-CTP-2531, to appear.

19. G. Barnich, F. Brandt, and M. Henneaux, 'Local BRST cohomology in Einstein-YangMills theory', hep-th/9505173; 'Local BRST cohomology in the antifield formalism: I. General theorems', hep-th/9405109; 'Local BRST cohomology in the antifield formalism: II Application to Yang-Mills theory', hep-th/9405194.

20. T. Kugo and B. Zwiebach, Prog. Theo. Phys. 87 (1992) 801

21. O.Bergman and B.Zwiebach 'The dilaton theorem and closed string backgrounds' Nucl. Phys. B441 (1995) 76, hep-th/9411047.

22. L. Cornalba, Connections over spaces of conformal field theories: testing for integrability, M.S. Thesis, MIT, May 1994. 